\title{
New ideas on the Proterozoic-Early Palaeozoic evolution of NW Iberia: insights from $\mathrm{U}-\mathrm{Pb}$ detrital zircon ages
}

\author{
J. Fernández-Suárez ${ }^{\mathrm{a}, 1}$, G. Gutiérrez-Alonso b,* ${ }^{\text {, G.A. Jenner }}{ }^{\mathrm{a}}$, M.N. Tubrett ${ }^{\mathrm{a}}$ \\ ${ }^{a}$ Department of Earth Sciences, Memorial University of Newfoundland, St. John's, Canada NF A1B 3X5 \\ ${ }^{\mathrm{b}}$ Departamento de Geología, Universidad de Salamanca, 33708 Salamanca, Spain
}

\begin{abstract}
$\mathrm{U}-\mathrm{Pb}$ ages were obtained on single detrital zircon grains separated from six samples of Neoproterozoic and Lower Palaeozoic sedimentary and volcanosedimentary rocks from NW Iberia using the laser ablation microprobe-inductively coupled plasma mass spectrometry (LAM-ICP-MS) method. Precambrian greywackes yielded abundant zircons with Neoproterozoic (800 - 640 Ma) and Mesoproterozoic (0.9 - $1.2 \mathrm{Ga})$ ages, and a smaller proportion of Palaeoproterozoic (1.8-2 Ga) and Archaean zircons. Palaeozoic samples (Lower Cambrian and Ordovician) yielded abundant zircons with younger Neoproterozoic (ca. 550 and $620 \mathrm{Ma}$ ) and Mesoproterozoic (0.9 - $1.2 \mathrm{Ga})$ ages. Palaeoproterozoic (1.8 - $2 \mathrm{Ga}$ ) and Archaean zircons were also found. This data set, used in conjuction with previous paleogeographic and isotopic studies sheds new light on the Precambrian-early Palaeozoic evolution of NW Iberia and is consistent with the following sequence of events: (1) Early Cadomian-Avalonian subduction and arc construction (ca. $800-640 \mathrm{Ma}$ ). This magmatic episode created the main arc edifice (Avalonia); (2) full development of a back arc basin upon which the Neoproterozoic sediments were deposited (ca. 640 - 600 Ma). The combined U- Pb ages of detrital zircons and $\mathrm{Nd}$ isotopic features of these sedimenary rocks suggest that they were mostly shed from the main magmatic arc. On the basis of the presence of Grenvillian age detrital zircons with short waterborne transport before incorporation in the sediment, we propose that the basin was possibly located in a peri-Amazonian realm close to West Avalonian terranes. These basins were developed upon a cratonic basement that possibly involved both Grenvillian (ca. $0.9-1.2 \mathrm{Ga}$ ) and Transamazonian (ca. 1.9-2.1) igneous rocks. The reported zircon ages suggest a long-lived subduction, starting at ca. $800 \mathrm{Ma}$ and terminated by ca. 580 - 570 Ma with no geological record of a final collision event; (3) the continuation of extension gave rise to the undocking of Avalonia from the back-arc. Detrital zircon ages in Lowermost Cambrian strata suggest that the main arc edifice had drifted away by ca. 550 - 540 Ma and was no longer shedding detritus into the back-arc basin. (4) During the Lower Ordovician, further extension of an already thinned crust gave rise to the Lower Ordovician 'Ollo de Sapo' magmatic event (ca. $480 \mathrm{Ma}$ ). Coeval volcanism in neighbouring areas displaying within-plate geochemical signatures is consistent with an extensional setting for the generation of the Lower Ordovician igneous and sedimentary rocks. Detrital zircon ages and $\mathrm{Nd}$
\end{abstract}

* Corresponding author. Tel.: + 34-923-294488; fax: +34-923-294514.

E-mail address: gabi@gugu.usal.es (G. Gutiérrez-Alonso)

${ }^{1}$ Present address: Departamento de Petrología y Geoquímica, Universidad Complutense, 28040 Madrid, Spain. 
isotopic features of the Ordovician greywackes reflect both an increase in the contribution from older crustal components and the addition of newly accreted crust. A progressively thinning crust is a likely scenario that would explain the simultaneous exhumation of lower crustal (Grenvillian+ Transamazonian/Icartian) material and the generation of coeval magmatism. This latter scenario is consistent with models proposed for other circum-North Atlantic Avalonian-Cadomian terranes where repeated episodes of melting occurred in response to subduction and subsequent rifting events.

Keywords: Iberia; Proterozoic; Early Palaeozoic; U-Pb; Laser ablation; Detrital zircons

\section{Introduction}

Knowledge of the nature, age and distribution of basement terranes in orogenic zones provides constraints on the evolution of igneous and sedimentary rocks formed during subsequent orogenic cycles. In areas where cratonic basement is not exposed, constraints on the ages and nature of older basement can be acquired by: presence of inherited zircons in magmatic rocks; detrital zircons in sedimentary rocks; and by neodymium isotopic studies. The significance and interpretation of these types of data are not always straightforward. For example, neodymium model ages/isotopic compositions of granitoids and detrital sedimentary rocks may not be interpretable without knowledge of the ages of crustal reservoirs that constituted sources for those granitoid melts or sedimentary detritus. Interpretation of detrital zircon ages can be hampered by uncertainties about transport distances (cf. Rainbird et al., 1997). Nonetheless, these types of studies provide data that, when used in conjunction with other geological criteria, are important in constraining the tectonic and paleogeographic evolution of ancient orogens.

The Variscan belt of Western Europe is a well known example of an orogenic belt lacking exposures of cratonic igneous or metamorphic basement. With the exception of the Palaeoproterozoic gneisses exposed in the Channel Islands (Calvez and Vidal, 1978; Samson and D’Lemos, 1998), little is known of the western European preNeoproterozoic basement. In the northwestern Iberian Massif (NW Iberia), thick Neoproterozoic successions of pelites and greywackes are the oldest exposed rocks and there is great uncertainty about the nature and age of the basement upon which they were deposited. NW Iberia is considered to have been part of the Neoproterozoic assemblage terranes (e.g. Nance and Murphy, 1996) that comprise the Cadomian-Avalonian magmatic arc and associated basins that developed on an active Gondwanan margin. Studies devoted to different aspects of the geological history of these terranes have provided indirect evidence for the existence of an older preNeoproterozoic basement at depth (e.g. Liew and Hofmann, 1988; Nägler et al., 1995; Nance and Murphy, 1996; Keppie et al., 1998). Direct evidence is, however, rather scarce (e.g. data compilation in Nance and Murphy, 1996; van Staal et al., 1996; Keppie et al., 1998; Samson and D’Lemos, 1998; Sheridan et al., 1999). In NW Iberia, there is some $\mathrm{U}-\mathrm{Pb}$ zircon evidence for Archaean (ca. 2.7 $\mathrm{Ga}$ ) and Palaeoproterozoic (ca. 1.9 Ga) crustal components (Guerrot et al., 1989; Gebauer, 1993). Furthermore, the neodymium isotopic composition of Neoproterozoic to Carboniferous sedimentary rocks and Cadomian and Variscan granitoids requires at least some component derived from Mesoproterozoic or older rocks (e.g. Beetsma, 1995; Nägler et al., 1995; MorenoVentas et al., 1995; Fernández-Suárez et al., 1998).

In order to place constraints on the ProterozoicEarly Palaeozoic evolution of NW Iberia and to better integrate this area in paleogeographic reconstructions of circum-Atlantic Gondwanan terranes, we have undertaken a study of the $\mathrm{U}-\mathrm{Pb}$ ages of detrital zircons obtained from Neoproterozoic, Cambrian and Ordovician sedimentary and volcanosedimentary rocks. These data were obtained by laser ablation microprobe-inductively coupled plasma-mass spectrometry (LAMICP-MS). The $\mathrm{U}-\mathrm{Pb}$ ages reported here have significant implications that may inspire further work and discussion on the role of Iberia in 
Avalonian-Cadomian and subsequent early Palaeozoic events.

\section{Geological and tectonic framework}

The autochthon of the Iberian Variscan belt (IVB) in NW Iberia is a well known example of a Palaeozoic collisional orogen (ca. 365 - 280 Ma) and has been extensively studied (Pérez-

Estaún et al., 1991; Martínez Catalán et al., 1996, 1997; Dallmeyer et al., 1997; and references therein). The NW Iberian Variscan belt (Fig. 1) has been divided into four main paleogeographic zones each consisting of sedimentary and igneous rocks of Neoproterozoic and Palaeozoic age. The differ- ent zones have a common Variscan tectonic evo- lution with varying styles of deformation, and increasing metamorphic grade and abundance of granitoids from the foreland (Cantabrian Zone) towards the suture. Prior to the Variscan evolu- tion, there is a record of late Cambrian and early Ordovician magmatic and tectonic events. Ob- scured by these Palaeozoic events, the Precam- brian evolution of NW Iberia is still poorly constrained.

An important feature of the autochthonous units of the IVB is the existence of large exposures of Neoproterozoic pelite-greywacke sedimentary sequences (Fig. 1). These rocks are exposed at antiformal culminations in the Narcea Antiform (Cantabrian Zone- West Asturian Leonese Zone limit), the Lugo Dome (West Asturian Leonese Zone) and in the Central Iberian Zone (Fig. 1). The Neoproterozoic sedimentary rocks are interpreted to have been deposited in an extensional basin generated during the Cadomian orogeny (Vidal et al., 1994). The basement upon which these rocks were deposited is not exposed anywhere in the autochthonous zone of the IVB.

In the Narcea Antiform (Fig. 1) two Neoproterozoic series of clastic sedimentary rocks (lithic arenites, greywackes and shales) have been distinguished: a lower series with abundant volcanics and intruded by scarce granitoids (Tineo Series, Gutiérrez-Alonso and Fernández-Suárez, 1996); and an upper series dominated by pelitic rocks with rare volcanics. In the Lugo dome (Fig.
1) the Lower and Upper Villalba Series have the same characteristics as their counterparts in the Narcea Antiform, except for a higher metamorphic grade (low- to medium-grade) (Capdevila, 1969; Martínez Catalán et al., 1996). The ca. 580 - 600 Ma granitoids (Fig. 1) (FernándezSuárez et al., 1998) intruding the Tineo Series provide a lower age limit for the deposition of these rocks, for which there is otherwise poor age control. Equivalent rocks in the Central Iberian Zone are also intruded by ca. 580 - 600 Ma granitoids (Leterrier and Noronha, 1998; Valle et al., 1999).

The uppermost Proterozoic-Lower Cambrian (Crimes et al., 1977) Cándana Formation unconformably overlays the Neoproterozoic rocks (Fig. 1 ) and is composed of quartzites, quartz-arenites, greywackes, arkoses and shales, interpreted to have been deposited in a braided-plain delta environment. Paleocurrents indicate that the provenance of the sediments is from the East- North East (van den Bosch, 1969; Crimes et al., 1977). In the Cantabrian and West Asturian Leonese zones, the Cándana Formation is overlain by a continuous Cambro-Ordovician passive continental margin sedimentary sequence. In the easternmost Central Iberian Zone, the Ordovician 'Ollo de Sapo complex', which includes both volcanosedimentary and magmatic rocks occurs in place of the passive continental margin sequence (Fig. 1). U- $\mathrm{Pb}$ geochronological data indicate Lower Ordovician ages for the magmatic rocks in the OS (Gebauer, 1993; Valverde-Vaquero and Dunning, 1997). The structure and origin of the OS is currently under debate and several hypothesis have been proposed (Capdevila, 1969; Martínez Catalán et al., 1997; Bastida et al., 1984; Ortega et al., 1996; Valverde-Vaquero and Dunning, 1997, 1999; Fernández-Suárez et al., 1998, 1999).

\section{Samples and zircon morphology}

Sample locations are shown in Fig. 1 and their stratigraphic position is schematically shown in the simplified columns of Fig. 2. The three Neoproterozoic rocks sampled for this study are 


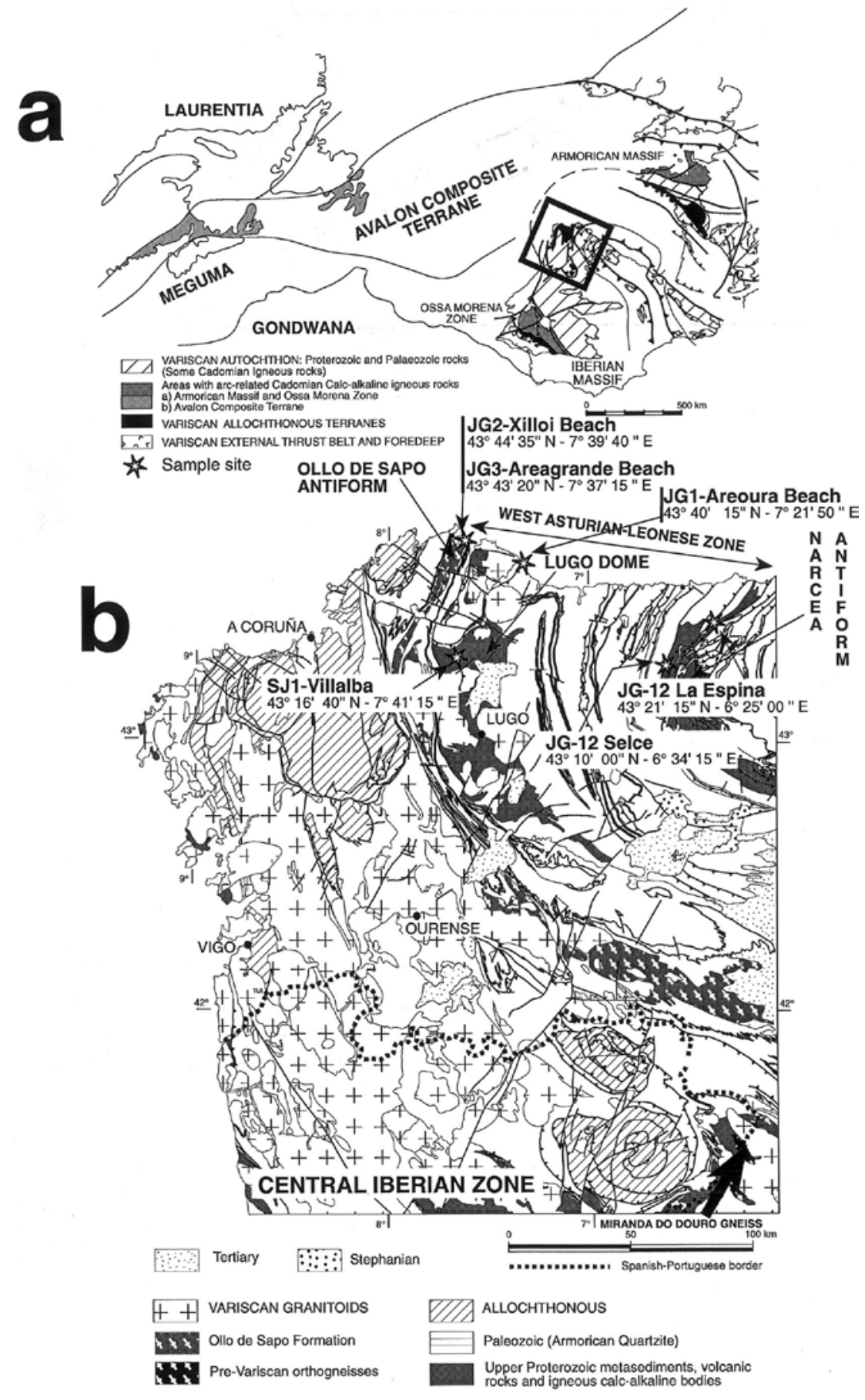

Fig. 1. (a) Reconstruction of Pangea around Iberia depicting the Ibero-Armorican Arc and the situation of the Avalon and Meguma terranes (after Martínez Catalán et al., 1997). (b) Geological map of part of the NW Iberian Variscan belt showing the main units, tectonic features and granitoids. Location of samples collected for this study is also indicated. 
metamorphosed (low-grade) medium-grained feldspathic greywackes from the Tineo Series (JG-12), Lower Villalba Series (JG-1) and Upper Villalba Series (SJ-1). Zircons separated from these samples are very similar in morphology, with a predominance of subhedral (facetted to subfacetted) long prismatic, tabular or stubby prisms (Fig. 3). This suggests that sediments are proximal to their source and that these zircons are unlikely to have been involved in more than one sedimentary cycle.

The Cambrian sample (JG-16) is a medium grained sub-arkose collected at the lower part of the Cándana Formation, approximately $10 \mathrm{~m}$ above the unconformity with the Tineo-Narcea Series. Zircons separated from this sample (Fig. 3) are yellow to light pink subhedral prismatic crystals also suggesting a short sedimentary transport. A small proportion of zircons were transparent sub-rounded grains with a size of less than ca. 30 microns which renders them unsuitable for LAM-ICP-MS dating (see below).

The two Ordovician samples are coarse- (JG2), and medium- (JG3) grained feldspathic greywackes. These rocks have been interpreted as immature sediments containing significant amounts of re-deposited volcanic component (Ortega et al., 1996 and references therein). Mineralogical and textural features such as poor sorting, high amount of matrix, occurrence of euhedral-subhedral quartz and feldspars, embayed quartz and zoned plagioclase suggest that: (i) these rocks were derived mainly from erosion of igneous sources (Capdevila, 1969; Ortega et al., 1996); and (ii) the sedimentary transport distance was short. Most zircons separated from the greywackes (and all the grains analysed) were euhedral to subhedral long to short prismatic or tabular suggesting a short transport and the improbability of their having undergone more than one sedimentary cycle.

Based on the immature (proximal) nature of the sedimentary rocks and on the morphological features of the zircons, we consider that the studied samples are unlikely to contain recycled zircons of exotic provenance (i.e. very long sedimentary transport, cf. Rainbird et al., 1997). It is also important to note that our results show no apparent correlation between the morphology of zircon grains and their $\mathrm{U}-\mathrm{Pb}$ age.

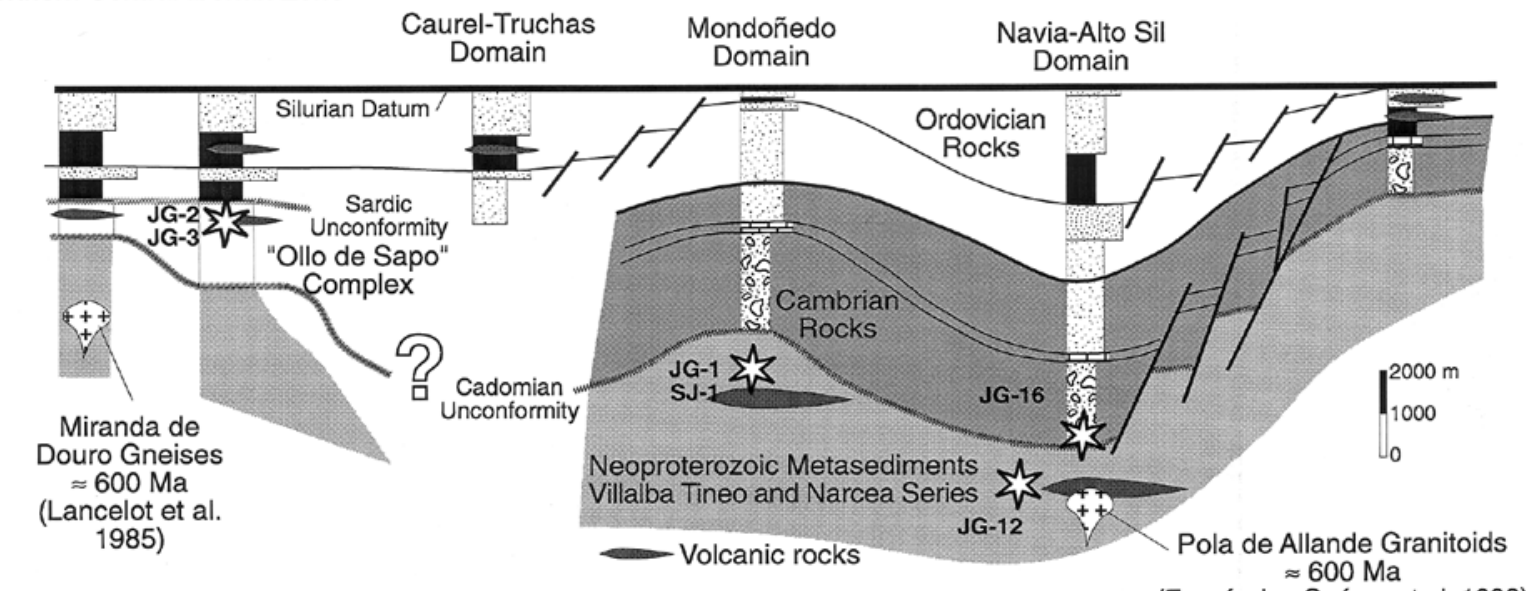

From Pérez-Estaún et al. 1990; Azor et

al. 1992 and Villar Alonso 1990

Fig. 2. Simplified stratigraphic column showing sample location in relation to the stratigraphic record. 

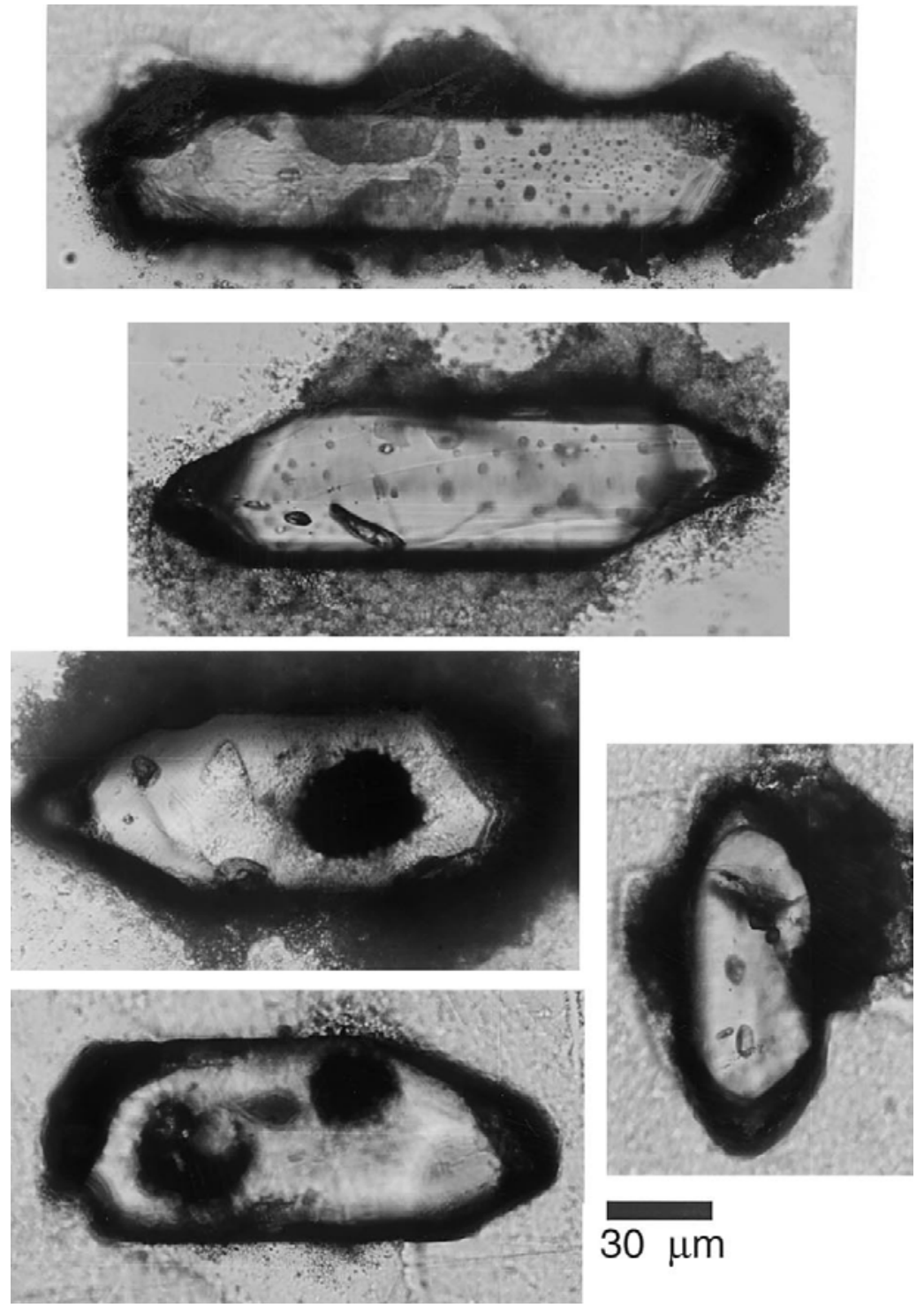

Fig. 3. Selected photographs of zircons from the studied samples.

\section{Sample preparation and analytical method}

Zircons were separated using conventional separation techniques (Wilfley table, heavy liquids and magnetic separation). Hand picked zircon grains were mounted in epoxy blocks, then polished to obtain an even surface, and cleaned in an acid bath prior to analysis. The LAM ICP-MS instrumentation, analytical conditions and data reduction software, were developed at Memorial University of Newfoundland and are partially described in Jackson et al. (1997). The instrumentation consists of an in-house built laser ablation microprobe incorporating a frequency quadrupled $\mathrm{Nd}$ :YAG laser (,= $266 \mathrm{~nm}$ in the UV). Ablated material is analysed by an enhanced sensitivity ICP-MS (FISONS VG PQII+'S'). This instrumentation has low ppb detection limits for $\mathrm{U}$ and 
$\mathrm{Pb}$ at $30-50$ micron sampling resolution. Ablation of the zircons is accompanied by some elemental fractionation so to minimize this we: (a) use a defocused laser beam to reduce thermal edge effects; (b) direct a jet of cooling Ar at the ablation point; and (c) use identical ablation conditions on the sample and standard (including the interval integrated for each analysis). Calibration is made using an in-house concordant zircon standard (Oslo Rift 02123 @ 295 \pm 1 Ma by thermal ionization mass spectrometry). Three measurements on the zircon standard and one on NBS 612 glass are made at the beginning and end of each run of 20 measurements. High instrumental $\mathrm{Hg}$ background prohibits accurate measurement of ${ }^{204} \mathrm{~Pb}$, so no common $\mathrm{Pb}$ correction is made. $\mathrm{Pb}$ background is minimised by careful attention to system cleanliness, and monitored throughout a run. Data are acquired in peak jumping mode using time-resolved software. Each analysis requires about $60 \mathrm{~s}$ of background, followed by $10-40 \mathrm{~s}$ of ablation depending on the sample size. The time-resolved signal data is reduced off-line, where it is possible to see the change in isotopic ratio profiles to detect zoning, and/or heterogeneities. Appropriate signal intervals are then integrated accordingly. ${ }^{235} \mathrm{U}$ counts are calculated from ${ }^{238} \mathrm{U}$. The strategies described allow the ratios of ${ }^{206} \mathrm{~Pb} /{ }^{238} \mathrm{U},{ }^{207} \mathrm{~Pb} /{ }^{235} \mathrm{U}$, and ${ }^{208} \mathrm{~Pb} /{ }^{232} \mathrm{Th}$ to be measured with precision (1 r.s.d.) of about $\pm 5 \%$ and ${ }^{207} \mathrm{~Pb} /{ }^{206} \mathrm{~Pb}$ to $\pm 1 \%$. Two sigma uncertainties on the ages determined using the individual ratios are given in Table 1. Accuracy using these procedures is estimated to be $\pm 10-20 \mathrm{Ma}$ based on comparing TIMS analyses of concordant zircons ranging from ca. 290-2700 Ma, with those obtained by LAM-ICP-MS on zircons from the same sample (sometimes the same grain) (Jackson and Dunning, unpublished).

\section{Data presentation and $\mathrm{U}-\mathrm{Pb}$ results}

LAM-ICP-MS is a relatively new technique and there is little background concerning the interpretation, discussion and presentation of analytical data, in comparison with the large amount of studies based on ion microprobe (SHRIMP,
SIMS) $\mathrm{U}-\mathrm{Pb}$ single zircon dating. In the latter case, results of $\mathrm{U}-\mathrm{Pb}$ analyses are currently reported in different ways such as Tera-Wasserburg $\left({ }^{207} \mathrm{~Pb} /{ }^{206} \mathrm{~Pb}\right.$ vs $\left.{ }^{238} \mathrm{U} /{ }^{206} \mathrm{~Pb}\right)$ concordia diagrams, conventional $\left({ }^{206} \mathrm{~Pb} /{ }^{238} \mathrm{U}\right.$ vs $\left.{ }^{207} \mathrm{~Pb} /{ }^{235} \mathrm{U}\right)$ concordia plots, age histograms and cumulative probability plots. A common feature to these techniques, in contrast to single grain TIMS $\mathrm{U}-\mathrm{Pb}$ analyses, are the data reduction strategies used (cf. Hirata and Nesbitt, 1995) and the assumptions made for isotopic ratio corrections. The above, combined with a lower capacity for isotope mass separation and measurement, results in less precise data and sometimes yields discordant data points that are not interpretable in a straightforward manner. In the present study, which reports a set of 83 zircon U- Th- Pb LAM-ICP-MS analyses with varying degrees of concordancy and precision, our major aim has been to identify the modes in the age spectra. We have been able to use the geological constraints on the data as a means for testing the meaningfulness of actual ages outside of the purely analytical interpretation. In addition, data sets were acquired on different selections of zircons in a number of samples, over a period of time, by three different analysts. These data sets all yielded consistent results. These analytical results are discussed in the following sections.

The analytical data containing isotope ratios, ages and errors are reported in Table 1 . We report $\mathrm{Pb} /{ }^{238} \mathrm{U} /{ }^{207} \mathrm{~Pb} /{ }^{235} \mathrm{U}$ concordant analyses on conventional concordia plots (Fig. 4). These plots were made using an unpublished program from the Royal Ontario Museum. In addition, a frequency histogram of ${ }^{207} \mathrm{~Pb} /{ }^{206} \mathrm{~Pb}$ ages (Fig. 5) is presented, that includes data from concordant and non-concordant analyses. We now briefly comment upon the criteria followed to select the best age estimate when all analyses are not concordant within error limits. For younger zircons in the age range from about 500 to $800 \mathrm{Ma}$, the ${ }^{206} \mathrm{~Pb} /{ }^{238} \mathrm{U}$ ages are preferred because ${ }^{207} \mathrm{~Pb} /{ }^{206} \mathrm{~Pb}$ ages are compromised by low ${ }^{207} \mathrm{~Pb}$ count rates. In these younger zircons, ${ }^{208} \mathrm{~Pb} /{ }^{232} \mathrm{Th}$ ages (Table 1) are often consistent with the ${ }^{206} \mathrm{~Pb} /{ }^{238} \mathrm{U}$ age. In discordant zircons older than ca. $1.5 \mathrm{Ga}$, we use ${ }^{207} \mathrm{~Pb} /{ }^{206} \mathrm{~Pb}$ ages because they have comparatively 
Table 1

LAM-ICP-MS U-Pb results

\begin{tabular}{|c|c|c|c|c|c|c|c|c|c|c|c|c|}
\hline \multirow[t]{2}{*}{ Analysis } & \multirow{2}{*}{$\begin{array}{l}{ }^{207} \mathrm{~Pb} /{ }^{206} \mathrm{~Pb} \\
\text { ratio }\end{array}$} & \multirow{2}{*}{$\begin{array}{l}{ }^{208} \mathrm{~Pb} /{ }^{232} \mathrm{Th} \\
\text { ratio }\end{array}$} & \multirow{2}{*}{$\begin{array}{l}{ }^{207} \mathrm{~Pb} /{ }^{235} \mathrm{U} \\
\text { ratio }\end{array}$} & \multirow{2}{*}{$\begin{array}{l}{ }^{206} \mathrm{~Pb} /{ }^{238} \mathrm{U} \\
\text { ratio }\end{array}$} & \multicolumn{2}{|l|}{${ }^{207} \mathrm{~Pb} /{ }^{235} \mathrm{U}$} & \multicolumn{2}{|l|}{${ }^{206} \mathrm{~Pb} /{ }^{238} \mathrm{U}$} & \multicolumn{2}{|l|}{${ }^{207} \mathrm{~Pb} /{ }^{206} \mathrm{~Pb}$} & \multicolumn{2}{|l|}{${ }^{208} \mathrm{~Pb} /{ }^{232} \mathrm{Th}$} \\
\hline & & & & & Age (Ma) & 2 & Age (Ma) & 2 & Age (Ma) & 2 & Age (Ma) & 2 \\
\hline \multicolumn{13}{|c|}{ Sample JG1 } \\
\hline $\mathrm{Z1}$ & 0.06422 & 0.04784 & 1.1732 & 0.1320 & 788 & 42 & 802 & 18 & 748 & 106 & 945 & 93 \\
\hline $\mathrm{Z} 2$ & 0.06666 & 0.06150 & 1.1587 & 0.1261 & 781 & 84 & 765 & 12 & 810 & 210 & 807 & 150 \\
\hline Z3 & 0.06616 & 0.04297 & 1.1005 & 0.1206 & 754 & 36 & 734 & 15 & 810 & 120 & 820 & 35 \\
\hline $\mathrm{Z} 4$ & 0.06187 & 0.03790 & 1.0523 & 0.1234 & 730 & 50 & 750 & 24 & 670 & 144 & 752 & 90 \\
\hline $\mathrm{Z} 5$ & 0.07133 & 0.04990 & 1.6029 & 0.1630 & 971 & 96 & 973 & 29 & 966 & 248 & 984 & 71 \\
\hline Z6 & 0.07472 & 0.05445 & 1.7730 & 0.1721 & 1036 & 67 & 1024 & 24 & 1060 & 108 & 1072 & 71 \\
\hline $\mathrm{Z7}$ & 0.06781 & 0.05985 & 1.4735 & 0.1576 & 920 & 55 & 943 & 38 & 870 & 90 & 1175 & 55 \\
\hline Z8 & 0.19366 & 0.11707 & 9.2877 & 0.3478 & 2367 & 81 & 1924 & 98 & 2772 & 152 & 2238 & 200 \\
\hline Z9 & 0.07124 & 0.04059 & 1.3420 & 0.1366 & 864 & 49 & 826 & 44 & 964 & 170 & 904 & 83 \\
\hline Z10 & 0.06973 & 0.05943 & 1.7695 & 0.1841 & 1034 & 81 & 1089 & 61 & 920 & 218 & 1168 & 78 \\
\hline Z11 & 0.07608 & 0.04474 & 1.5921 & 0.1518 & 967 & 55 & 911 & 42 & 1096 & 198 & 885 & 117 \\
\hline Z12 & 0.06392 & 0.02684 & 0.8283 & 0.0940 & 613 & 50 & 579 & 52 & 738 & 134 & 685 & 62 \\
\hline Z13 & 0.07005 & 0.04998 & 1.7446 & 0.1806 & 1025 & 44 & 1070 & 48 & 928 & 90 & 986 & 53 \\
\hline Z14 & 0.06762 & 0.04838 & 1.4920 & 0.1600 & 927 & 50 & 957 & 55 & 856 & 126 & 955 & 40 \\
\hline Z15 & 0.06310 & 0.03606 & 1.1159 & 0.1283 & 761 & 36 & 778 & 20 & 710 & 160 & 716 & 14 \\
\hline Z16 & 0.06765 & 0.04712 & 1.4536 & 0.1558 & 911 & 47 & 934 & 57 & 856 & 112 & 931 & 59 \\
\hline Z17 & 0.06453 & 0.03107 & 1.0206 & 0.1147 & 714 & 59 & 700 & 63 & 758 & 154 & 618 & 53 \\
\hline Z18 & 0.10063 & 0.07156 & 2.6214 & 0.1889 & 1307 & 29 & 1116 & 7 & 1634 & 64 & 1397 & 49 \\
\hline \multicolumn{13}{|c|}{ Sample SJ1 } \\
\hline $\mathrm{Z1}$ & 0.07324 & 0.05062 & 1.6371 & 0.1621 & 985 & 30 & 968 & 24 & 1020 & 84 & 998 & 22 \\
\hline $\mathrm{Z} 2$ & 0.07135 & 0.03451 & 1.1868 & 0.1206 & 794 & 67 & 734 & 26 & 966 & 292 & 686 & 37 \\
\hline Z3 & 0.06853 & 0.02844 & 1.0489 & 0.1110 & 728 & 47 & 679 & 13 & 884 & 178 & 667 & 32 \\
\hline $\mathrm{Z} 4$ & 0.08298 & 0.06352 & 2.1493 & 0.1879 & 1165 & 22 & 1110 & 13 & 1268 & 70 & 1245 & 55 \\
\hline Z5 & 0.07521 & 0.04585 & 1.5077 & 0.1454 & 934 & 21 & 875 & 16 & 1074 & 72 & 926 & 18 \\
\hline Z6 & 0.06233 & 0.03150 & 0.8950 & 0.1041 & 649 & 39 & 639 & 19 & 684 & 176 & 627 & 33 \\
\hline $\mathrm{Z7}$ & 0.18392 & 0.13036 & 10.7787 & 0.4250 & 2504 & 26 & 2283 & 61 & 2688 & 50 & 2477 & 44 \\
\hline Z8 & 0.18117 & 0.11694 & 9.3416 & 0.3740 & 2372 & 33 & 2048 & 62 & 2662 & 46 & 2235 & 51 \\
\hline Z9 & 0.16074 & 0.09445 & 6.7988 & 0.3066 & 2086 & 34 & 1724 & 71 & 2462 & 40 & 1824 & 137 \\
\hline Z10 & 0.12238 & 0.08180 & 4.5926 & 0.2720 & 1748 & 37 & 1551 & 29 & 1990 & 94 & 1589 & 30 \\
\hline Z11 & 0.07437 & 0.03050 & 0.9333 & 0.0910 & 669 & 44 & 561 & 36 & 1050 & 146 & 607 & 55 \\
\hline Z12 & 0.06495 & 0.02268 & 0.6997 & 0.0781 & 539 & 26 & 485 & 9 & 772 & 140 & 753 & 36 \\
\hline Z13 & 0.06378 & 0.02448 & 0.6776 & 0.0770 & 525 & 21 & 478 & 8 & 734 & 110 & 789 & 27 \\
\hline Z14 & 0.08931 & 0.04848 & 1.8428 & 0.1496 & 1061 & 60 & 899 & 31 & 1410 & 140 & 957 & 63 \\
\hline Z15 & 0.06759 & 0.03317 & 1.1645 & 0.1249 & 784 & 26 & 759 & 12 & 854 & 92 & 660 & 40 \\
\hline Z16 & 0.07406 & 0.05227 & 1.7962 & 0.1758 & 1044 & 59 & 1044 & 77 & 1042 & 82 & 1030 & 127 \\
\hline Z17 & 0.13953 & 0.09612 & 3.0919 & 0.1607 & 1431 & 86 & 960 & 46 & 2220 & 190 & 1855 & 243 \\
\hline Z18 & 0.07752 & 0.06718 & 2.2928 & 0.2145 & 1210 & 45 & 1253 & 57 & 1134 & 82 & 1314 & 72 \\
\hline Z19 & 0.06715 & 0.03946 & 1.1057 & 0.1194 & 756 & 40 & 727 & 36 & 842 & 122 & 782 & 61 \\
\hline
\end{tabular}


Table 1 (Continued)

\begin{tabular}{|c|c|c|c|c|c|c|c|c|c|c|c|c|}
\hline \multirow[t]{2}{*}{ Analysis } & \multirow{2}{*}{$\begin{array}{l}{ }^{207} \mathrm{~Pb} /{ }^{206} \mathrm{~Pb} \\
\text { ratio }\end{array}$} & \multirow{2}{*}{$\begin{array}{l}{ }^{208} \mathrm{~Pb} /{ }^{232} \mathrm{Th} \\
\text { ratio }\end{array}$} & \multirow{2}{*}{$\begin{array}{l}{ }^{207} \mathrm{~Pb} /{ }^{235} \mathrm{U} \\
\text { ratio }\end{array}$} & \multirow{2}{*}{$\begin{array}{l}{ }^{206} \mathrm{~Pb} /{ }^{238} \mathrm{U} \\
\text { ratio }\end{array}$} & \multicolumn{2}{|l|}{${ }^{207} \mathrm{~Pb} /{ }^{235} \mathrm{U}$} & \multicolumn{2}{|l|}{${ }^{206} \mathrm{~Pb} /{ }^{238} \mathrm{U}$} & \multicolumn{2}{|l|}{${ }^{207} \mathrm{~Pb} /{ }^{206} \mathrm{~Pb}$} & \multicolumn{2}{|l|}{${ }^{208} \mathrm{~Pb} /{ }^{232} \mathrm{Th}$} \\
\hline & & & & & Age (Ma) & 2 & Age (Ma) & 2 & Age (Ma) & 2 & Age (Ma) & 2 \\
\hline \multicolumn{13}{|c|}{ Sample JG12 } \\
\hline $\mathrm{Z1}$ & 0.06086 & 0.03637 & 0.9072 & 0.1081 & 656 & 55 & 662 & 32 & 634 & 146 & 722 & 49 \\
\hline $\mathrm{Z} 2$ & 0.06326 & 0.03552 & 0.9887 & 0.1133 & 698 & 19 & 692 & 17 & 716 & 112 & 705 & 14 \\
\hline $\mathrm{Z3}$ & 0.07485 & 0.04627 & 1.6027 & 0.1553 & 971 & 56 & 931 & 22 & 1064 & 62 & 994 & 68 \\
\hline Z4 & 0.07859 & 0.05579 & 1.6059 & 0.1482 & 972 & 97 & 899 & 80 & 1160 & 192 & 1097 & 74 \\
\hline Z5 & 0.06443 & 0.04122 & 1.0360 & 0.1166 & 722 & 61 & 711 & 27 & 754 & 236 & 816 & 121 \\
\hline Z6 & 0.11505 & 0.08049 & 4.7499 & 0.2994 & 1776 & 37 & 1688 & 35 & 1880 & 90 & 1690 & 22 \\
\hline Z7 & 0.06463 & 0.03994 & 1.1580 & 0.1300 & 758 & 91 & 788 & 14 & 650 & 290 & 781 & 33 \\
\hline Z8 & 0.11257 & 0.12303 & 5.1013 & 0.3287 & 1836 & 65 & 1832 & 55 & 1840 & 146 & 1945 & 111 \\
\hline Z9 & 0.07376 & 0.05312 & 1.8595 & 0.1828 & 1067 & 83 & 1082 & 32 & 1034 & 230 & 1046 & 88 \\
\hline Z10 & 0.09780 & 0.07265 & 2.5323 & 0.1878 & 1281 & 87 & 1109 & 52 & 1582 & 198 & 1481 & 119 \\
\hline Z11 & 0.07196 & 0.04886 & 1.6706 & 0.1684 & 997 & 90 & 1003 & 74 & 984 & 152 & 964 & 145 \\
\hline Z12 & 0.06624 & 0.04231 & 1.2514 & 0.1370 & 824 & 35 & 828 & 23 & 814 & 86 & 838 & 39 \\
\hline Z13 & 0.08740 & 0.07322 & 2.5964 & 0.2154 & 1300 & 73 & 1260 & 56 & 1358 & 224 & 1228 & 120 \\
\hline \multicolumn{13}{|c|}{ Sample JG16 } \\
\hline $\mathrm{Z1}$ & 0.05531 & 0.02567 & 0.6758 & 0.0886 & 524 & 60 & 547 & 17 & 484 & 228 & 512 & 32 \\
\hline $\mathrm{Z} 2$ & 0.07170 & 0.02782 & 0.9208 & 0.0931 & 663 & 96 & 574 & 29 & 976 & 206 & 555 & 74 \\
\hline $\mathrm{Z3}$ & 0.06329 & 0.03176 & 0.8854 & 0.1014 & 644 & 73 & 623 & 22 & 718 & 270 & 632 & 63 \\
\hline Z4 & 0.16592 & 0.10206 & 7.9378 & 0.3470 & 2224 & 101 & 1920 & 86 & 2516 & 208 & 1964 & 211 \\
\hline Z5 & 0.15332 & 0.10491 & 7.8107 & 0.3695 & 2209 & 26 & 2027 & 22 & 2382 & 48 & 2017 & 51 \\
\hline Z6 & 0.06604 & 0.02785 & 0.8266 & 0.09098 & 612 & 68 & 560 & 28 & 806 & 294 & 573 & 45 \\
\hline Z7 & 0.07302 & 0.04098 & 1.3646 & 0.1355 & 874 & 62 & 819 & 28 & 1014 & 100 & 972 & 83 \\
\hline Z8 & 0.17240 & 0.10083 & 9.3368 & 0.3928 & 2372 & 46 & 2136 & 52 & 2580 & 60 & 1942 & 54 \\
\hline Z9 & 0.06020 & 0.02688 & 0.7513 & 0.0905 & 569 & 34 & 559 & 16 & 610 & 186 & 536 & 31 \\
\hline Z10 & 0.06545 & 0.02891 & 0.8248 & 0.0985 & 611 & 20 & 600 & 8 & 620 & 94 & 576 & 54 \\
\hline Z11 & 0.06764 & 0.03715 & 1.1561 & 0.1240 & 780 & 31 & 753 & 21 & 856 & 114 & 737 & 41 \\
\hline Z12 & 0.07296 & 0.04341 & 1.4440 & 0.1435 & 907 & 81 & 865 & 30 & 1012 & 258 & 959 & 38 \\
\hline Z13 & 0.07613 & 0.03344 & 1.2212 & 0.1163 & 810 & 52 & 709 & 23 & 1098 & 174 & 995 & 34 \\
\hline Z14 & 0.06097 & 0.02640 & 0.8266 & 0.0983 & 612 & 43 & 605 & 15 & 638 & 206 & 567 & 34 \\
\hline Z15 & 0.06445 & 0.02111 & 0.7679 & 0.0864 & 579 & 19 & 534 & 16 & 756 & 106 & 522 & 18 \\
\hline \multicolumn{13}{|c|}{ Sample JG2 } \\
\hline $\mathrm{Z1}$ & 0.11890 & 0.10200 & 5.0798 & 0.3537 & 1946 & 19 & 1952 & 24 & 1938 & 32 & 1963 & 30 \\
\hline $\mathrm{Z} 2$ & 0.07611 & 0.05145 & 1.9224 & 0.1832 & 1089 & 20 & 1084 & 14 & 1098 & 60 & 1014 & 92 \\
\hline $\mathrm{Z3}$ & 0.07478 & 0.05106 & 1.9251 & 0.1867 & 1090 & 16 & 1104 & 15 & 1062 & 54 & 1017 & 35 \\
\hline Z4 & 0.06047 & 0.02678 & 0.7694 & 0.0923 & 579 & 29 & 569 & 18 & 620 & 120 & 534 & 32 \\
\hline Z5 & 0.05605 & 0.01992 & 0.5507 & 0.0713 & 445 & 29 & 444 & 16 & 454 & 140 & 400 & 30 \\
\hline Z6 & 0.05418 & 0.01870 & 0.5315 & 0.0711 & 433 & 28 & 443 & 8 & 380 & 190 & 374 & 30 \\
\hline Z7 & 0.20557 & 0.010283 & 12.1646 & 0.4292 & 2617 & 18 & 2302 & 26 & 2840 & 24 & 1978 & 32 \\
\hline Z8 & 0.11769 & 0.07568 & 4.8652 & 0.2998 & 1796 & 24 & 1690 & 23 & 1920 & 52 & 1475 & 39 \\
\hline
\end{tabular}


Table 1 (Continued)

\begin{tabular}{|c|c|c|c|c|c|c|c|c|c|c|c|c|}
\hline \multirow[t]{2}{*}{ Analysis } & \multirow{2}{*}{$\begin{array}{l}{ }^{207} \mathrm{~Pb} /{ }^{206} \mathrm{~Pb} \\
\text { ratio }\end{array}$} & \multirow{2}{*}{$\begin{array}{l}{ }^{208} \mathrm{~Pb} /{ }^{232} \mathrm{Th} \\
\text { ratio }\end{array}$} & \multirow{2}{*}{$\begin{array}{l}{ }^{207} \mathrm{~Pb} /{ }^{235} \mathrm{U} \\
\text { ratio }\end{array}$} & \multirow{2}{*}{$\begin{array}{l}{ }^{206} \mathrm{~Pb} /{ }^{238} \mathrm{U} \\
\text { ratio }\end{array}$} & \multicolumn{2}{|l|}{${ }^{207} \mathrm{~Pb} /{ }^{235} \mathrm{U}$} & \multicolumn{2}{|l|}{${ }^{206} \mathrm{~Pb} /{ }^{238} \mathrm{U}$} & \multicolumn{2}{|l|}{${ }^{207} \mathrm{~Pb} /{ }^{206} \mathrm{~Pb}$} & \multicolumn{2}{|l|}{${ }^{208} \mathrm{~Pb} /{ }^{232} \mathrm{Th}$} \\
\hline & & & & & Age (Ma) & 2 & Age (Ma) & 2 & Age (Ma) & 2 & Age (Ma) & 2 \\
\hline Z9 & 0.07862 & 0.06650 & 1.5280 & 0.1410 & 942 & 48 & 850 & 47 & 1162 & 114 & 1301 & 107 \\
\hline \multicolumn{13}{|c|}{ Sample JG3 } \\
\hline $\mathrm{Z} 1$ & 0.12189 & 0.08705 & 5.9961 & 0.3568 & 1975 & 35 & 1967 & 37 & 1984 & 68 & 1687 & 67 \\
\hline $\mathrm{Z} 2$ & 0.12349 & 0.09507 & 5.7901 & 0.3353 & 1933 & 37 & 1864 & 31 & 2006 & 80 & 1836 & 58 \\
\hline Z3 & 0.07760 & 0.05865 & 2.1556 & 0.2015 & 1167 & 19 & 1183 & 12 & 1136 & 58 & 1152 & 48 \\
\hline $\mathrm{Z} 4$ & 0.06133 & 0.02819 & 0.8174 & 0.0967 & 607 & 21 & 595 & 9 & 650 & 98 & 562 & 16 \\
\hline $\mathrm{Z} 5$ & 0.058782 & 0.02916 & 0.7992 & 0.1002 & 596 & 12 & 616 & 7 & 522 & 56 & 581 & 11 \\
\hline Z6 & 0.05925 & 0.03633 & 0.7886 & 0.0965 & 590 & 36 & 594 & 30 & 576 & 100 & 546 & 40 \\
\hline $\mathrm{Z7}$ & 0.05440 & 0.02123 & 0.5546 & 0.0739 & 448 & 16 & 460 & 6 & 386 & 92 & 425 & 11 \\
\hline Z8 & 0.11200 & 0.10426 & 4.8299 & 0.31270 & 1790 & 32 & 1754 & 34 & 1832 & 54 & 2005 & 41 \\
\hline Z9 & 0.07669 & 0.06984 & 2.3241 & 0.2198 & 1220 & 24 & 1281 & 20 & 1112 & 64 & 1265 & 45 \\
\hline
\end{tabular}


smaller uncertainties owing to higher amounts of ${ }^{207} \mathrm{~Pb}$. In the case of zircons of intermediate age $(1-1.5 \mathrm{Ga}){ }^{207} \mathrm{~Pb} /{ }^{206} \mathrm{~Pb}$ age is preferred when it is

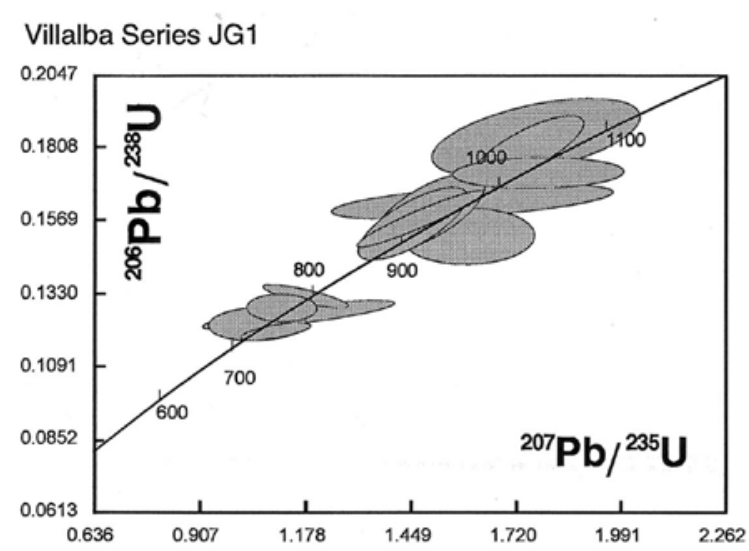

Tineo Series (Narcea

Slates) JG12

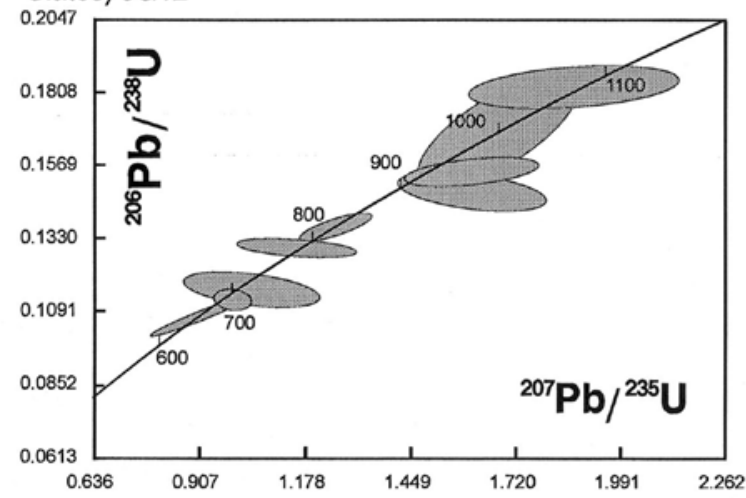

Ollo de Sapo Complex

JG2 and JG3

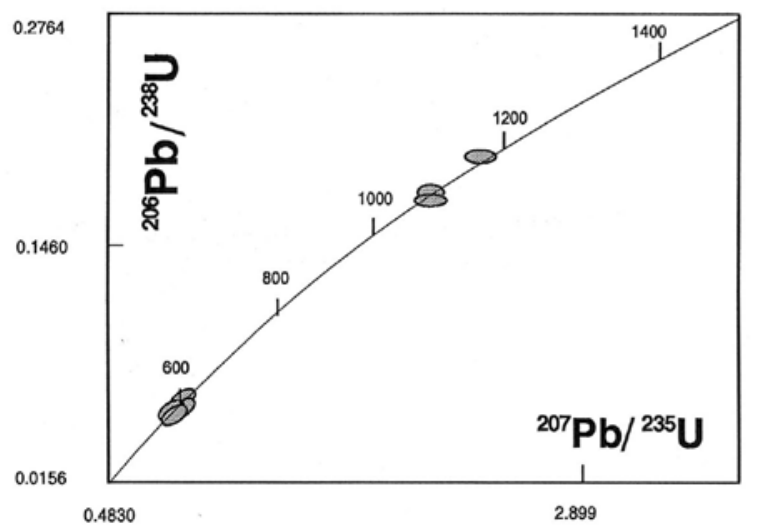

consistent with the ${ }^{208} \mathrm{~Pb} /{ }^{238} \mathrm{Th}$ age. The ${ }^{208} \mathrm{~Pb} /$ ${ }^{238} \mathrm{U}$ age is otherwise preferred. Similar weighting of young ages to ${ }^{206} \mathrm{~Pb} /{ }^{238} \mathrm{U}$ and older ages to

Villalba Series SJ1

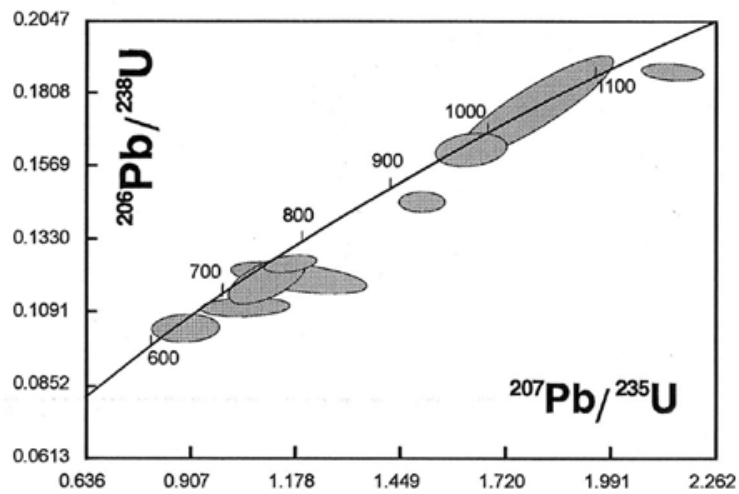

Cándana Series JG16
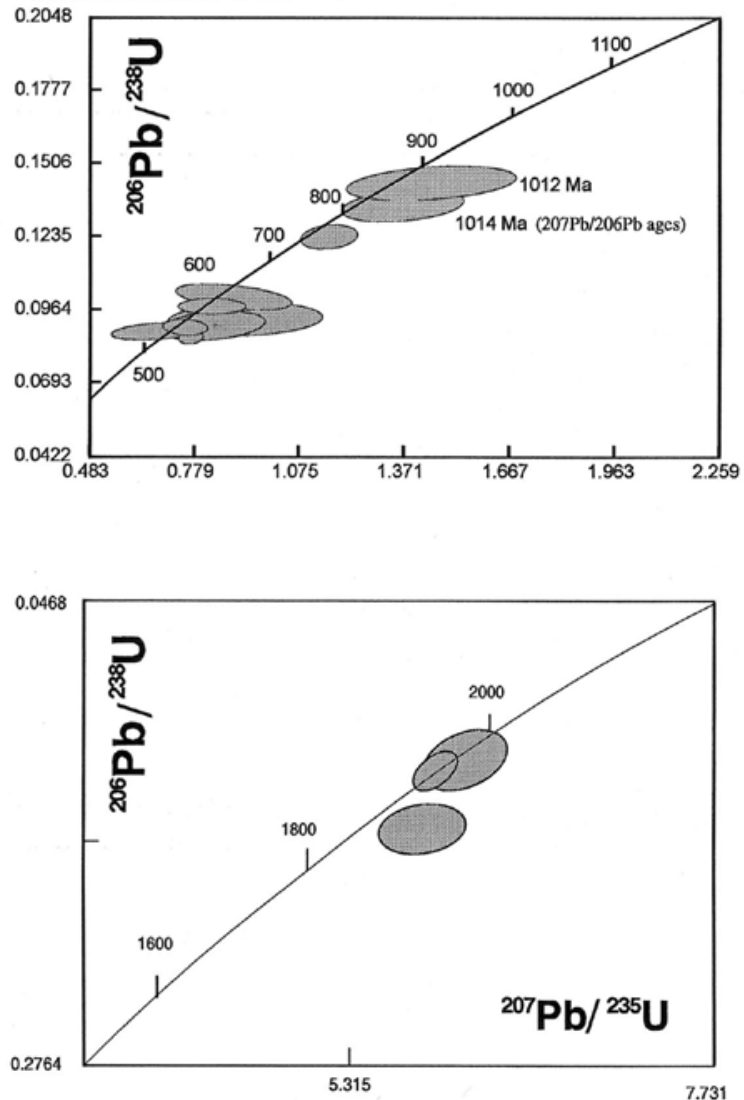

Fig. 4. Concordia plots of $\mathrm{U}-\mathrm{Pb}$ analytical data. Ellipses represent 2 uncertainties. 


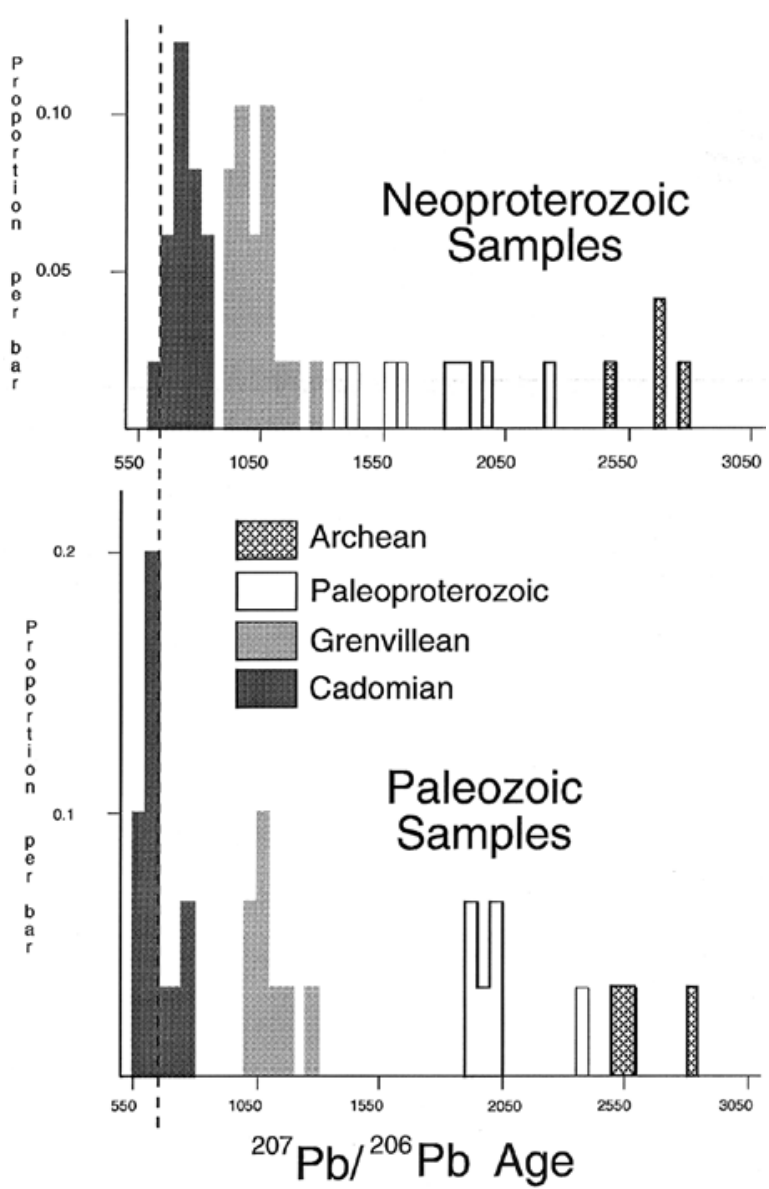

Fig. 5. Histograms of ${ }^{207} \mathrm{~Pb} /{ }^{206} \mathrm{~Pb}$ ages. (a) Neoproterozoic samples; (b) lower Palaeozoic samples.

${ }^{207} \mathrm{~Pb} /{ }^{206} \mathrm{~Pb}$ is used in ion probe work (cf. Ireland et al., 1998).

\subsection{Proterozoic metagreywackes (JG1, JG12 and} SJ1) (Fig. 4a, b, c)

Zircons in sample JG-1 (Lower Villalba Series) yielded a number of sub-concordant analyses with Neoproterozoic ${ }^{206} \mathrm{~Pb} /{ }^{238} \mathrm{U}$ ages ranging from ca. 800 to са. $700 \mathrm{Ma}$ and Middle Proterozoic ${ }^{206} \mathrm{~Pb} /$ ${ }^{238} \mathrm{U}$ ages ranging from 911 to $1089 \mathrm{Ma}$. Zircons Z8 and Z18 are discordant and have Achaean (ca. $2.77 \mathrm{Ga}$ ) and Palaeoproterozoic (ca. $1.63 \mathrm{Ga}$ ) ${ }^{207} \mathrm{~Pb} /{ }^{206} \mathrm{~Pb}$ ages. Z12 has a somewhat ambiguous age around $600 \mathrm{Ma}$, while Z9 is best described by an age of ca. 800-850 Ma.
Five zircons from sample SJ-1 (Upper Villalba Series) yielded Late Proterozoic subconcordant ages (Table 1, Fig. 4b) with ${ }^{206} \mathrm{~Pb} /{ }^{238} \mathrm{U}$ ages ranging from 639 to $759 \mathrm{Ma}$. Z12 and Z13 are discordant and yielded Neoproterozoic ${ }^{207} \mathrm{~Pb} /{ }^{206} \mathrm{~Pb}$ ages of 772 and $734 \mathrm{Ma}$, respectively. Note that Z12 is a rim to Z19. Zircons Z1, Z16 and Z18 are subconcordant and yielded Middle Proterozoic ${ }^{206} \mathrm{~Pb} /{ }^{238} \mathrm{U}$ ages of 968, 1044 and $1253 \mathrm{Ma}$, respectively. Zircons Z4, Z5 and Z11 are discordant and yielded Mesoproterozoic ${ }^{207} \mathrm{~Pb} /{ }^{206} \mathrm{~Pb}$ ages of 1268 , 1074 and $1050 \mathrm{Ma}$, respectively. Zircons Z10 and Z17 are discordant and yielded Palaeoproterozoic ${ }^{207} \mathrm{~Pb} /{ }^{206} \mathrm{~Pb}$ ages of 1990 and $2220 \mathrm{Ma}$, respectively. Discordant analysis Z14 yielded a late Mesoproterozoic ${ }^{207} \mathrm{~Pb} /{ }^{206} \mathrm{~Pb}$ age $(1410 \mathrm{Ma})$. Finally, discordant analyses Z7, Z8 and Z9 yielded Archaean ${ }^{207} \mathrm{~Pb} /{ }^{206} \mathrm{~Pb}$ ages between 2462 and 2688 Ma.

Five zircons from sample JG-12 (Tineo Series) yielded Late Proterozoic ages (Fig. 4c, Table 1), with ${ }^{206} \mathrm{~Pb} /{ }^{238} \mathrm{U}$ ages ranging from 662 to $828 \mathrm{Ma}$. Three zircons yielded concordant Mesoproterozoic ages with ${ }^{206} \mathrm{~Pb} /{ }^{238} \mathrm{U}$ ages of 1082,1003 and $1260 \mathrm{Ma}$, respectively. Z3 and Z4 yielded subconcordant data points with ${ }^{206} \mathrm{~Pb} /{ }^{238} \mathrm{U}$ ages of 931 and $900 \mathrm{Ma}$ and ${ }^{207} \mathrm{~Pb} /{ }^{206} \mathrm{~Pb}$ ages of 1064 and $1164 \mathrm{Ma}$, respectively. In this case, the ${ }^{207} \mathrm{~Pb} /{ }^{206} \mathrm{~Pb}$ ages are preferred as they are more consistent with the $\mathrm{Th} / \mathrm{Pb}$ ages (Table 1 ). Zircon $\mathrm{Z} 8$ yielded a concordant Palaeoproterozoic age of ca. 1.83 $\mathrm{Ga}\left({ }^{206} \mathrm{~Pb} /{ }^{238} \mathrm{U}\right.$ age) (not shown in Fig. 4). Zircons Z6 and Z10 are discordant and yielded Palaeoproterozoic ${ }^{207} \mathrm{~Pb} /{ }^{206} \mathrm{~Pb}$ ages of ca. 1.88 and $1.58 \mathrm{Ga}$, respectively.

\subsection{Cambrian sandstone (Cándana Formation, JG16)}

Eight zircons separated from the Lower Cambrian arkose yielded Late Proterozoic ages younger than those of zircons found in the Neoproterozoic greywackes, the ${ }^{206} \mathrm{~Pb} /{ }^{238} \mathrm{U}$ ages ranging from ca. 540 to ca. 623 Ma. Sub-concordant (Z7, Z12) and discordant (Z13) zircons yielded ${ }^{207} \mathrm{~Pb} /{ }^{206} \mathrm{~Pb}$ Mesoproterozoic ages of 1014 , 1012 and $1098 \mathrm{Ma}$, respectively. Although the uncertainties associated to the ${ }^{207} \mathrm{~Pb} /{ }^{206} \mathrm{~Pb}$ ages are 
quite large, these ages are in this case preferred to the $\mathrm{U}-\mathrm{Pb}$ ages since they are more consistent with the $\mathrm{Th} / \mathrm{Pb}$ ages (Table 1 ). In addition, three discordant zircons (Z4, Z5 and Z8) have ${ }^{207} \mathrm{~Pb} /$ ${ }^{206} \mathrm{~Pb}$ ages of ca. $2.4-2.6 \mathrm{Ga}$ (Archaean-Early Proterozoic boundary).

\subsection{Ordovician volcanosedimentary} metagreywackes (Ollo de Sapo complex, JG2 and JG3)

Zircons from these samples yielded mostly concordant $\mathrm{U}-\mathrm{Pb}$ ages that fall into four age groupings: Ordovician (ca. 440 - 460 Ma), Late Neoproterozoic (ca. 570-620), Mesoproterozoic (ca. 1-1.2 Ga) and Palaeoproterozoic (ca. 1.9-2 $\mathrm{Ga}$ ). The youngest $\mathrm{U}-\mathrm{Pb}$ age grouping (ca. $440-$ 460 , Table 1) is considered to represent the age of the volcanic component of the OS rocks. This age is younger than ages reported for other magmatic units of this complex in areas situated further to the S-SE along the OS antiform (ca. 465-490 Ma, Lancelot et al., 1985; Gebauer, 1993; ValverdeVaquero and Dunning, 1997). One zircon from sample JG2 (Z4) and three from sample JG-3 (Z4, Z5, Z6) yielded Late Proterozoic concordant ages (Fig. 4e). The ${ }^{206} \mathrm{~Pb} /{ }^{238} \mathrm{U}$ ages range from ca. 569 to ca. 616 Ma. The third group, of which three concordant ages are reported in Fig. 4e, represents the recycling of a mid-Proterozoic crustal component of ca. 1-1.2 Ga. Two zircons from sample JG-2 (Z2 and Z3) yielded overlapping concordant ages of ca. $1080-1105 \mathrm{Ma}$ and a zircon from sample JG3 (Z3) yielded an older concordant age of ca. 1183 Ma. Zircons Z9 (JG2) and Z9 (JG3) yielded discordant data points with ${ }^{207} \mathrm{~Pb} /{ }^{206} \mathrm{~Pb}$ ages of 1162 and $1112 \mathrm{Ma}$, respectively. The fourth group of recycled zircons is represented by two concordant and partially overlapping $\mathrm{U}-\mathrm{Pb}$ ages of ca. $1952 \mathrm{Ma}$ (Z1, sample JG2) and ca. 1967 Ma (Z1, sample JG3). A second zircon from JG3 (Z2), although not concordant, yielded a ${ }^{207} \mathrm{~Pb} /{ }^{206} \mathrm{~Pb}$ age of $2 \mathrm{Ga}$, within error limits of the concordant zircon from the same sample. Discordant analyses Z8 (JG2) and Z8 (JG3) yielded ${ }^{207} \mathrm{~Pb} /{ }^{206} \mathrm{~Pb}$ ages of 1920 and $1832 \mathrm{Ma}$, respectively. Finally, a discordant zircon from sample JG-2 (Z6) has a ${ }^{207} \mathrm{~Pb} /{ }^{206} \mathrm{~Pb}$ age of ca. $2.8 \mathrm{Ga}$, the oldest Archaean age found in this study.

\section{Discussion}

Before discussing the main implications of the ages reported in this study, we must consider how representative the samples analysed are. It is difficult to ascertain how many individual analyses might be required; for instance Ireland et al. (1998) use 50 zircons per sample. However, we consider that given: (a) the geological consistency of the data; (b) our own tests, wherein we analysed additional grains (eight to ten) on separate occasions but found no new age groups; and (c) the implications of actual ages and apparent age-group distribution; the results of this study provide sufficient grounds for a preliminary hypothesis on their geological implications.

\subsection{Comparison of zircon ages with tectonothermal and crustal growth events in Gondwana}

The $\mathrm{U}-\mathrm{Pb}$ ages of detrital zircons in Neoproterozoic and early Palaeozoic rocks from NW Iberia reveal a varied provenance including newly accreted crust and old cratonic crustal sources. The youngest zircon age population found in the Neoproterozoic greywackes (ca. $640-800 \mathrm{Ma}$ ) corresponds to the period of early arc magmatism in the peripheral orogens around Gondwana, in which subduction related magmatic activity began at ca. 800 Ma (e.g. Murphy and Nance, 1991). Although the most complete record of early arc activity is preserved in the Arabian shield (e.g. Krö ner et al., 1988), there is also a record of this early stage in the AvalonianCadomian belt (O’Brien et al., 1996; Egal et al., 1996). The data reported in this study suggest that the Neoproterozoic sedimentary rocks in NW Iberia contain a significant detrital component generated by erosion of igneous rocks formed in the early stages of the construction of the Cadomian-Avalonian magmatic arc. The data also bracket the deposition age of the Neoproterozoic sedimentary sequences between ca. 600 Ma (age 
of granitoids intruded into the Tineo Series, Fernández-Suárez et al., 1998) and ca. 640 Ma (age of the youngest detrital zircons found in these Formations). This age interval matches the period of full development of back-arc basins in the Avalon Composite Terrane (са. 630 - 610 Ma) proposed by Keppie et al. (1998).

Cambrian and Ordovician samples in contrast (Figs. 4 and 5 and Table 1) yielded very few zircons in the $640-800$ age group and instead contain abundant younger zircons (ca. 550-620 $\mathrm{Ma})$. This age interval corresponds to the crystallisation ages of Cadomian granitoids intruded into the Neoproterozoic sequences in NW Iberia (Lancelot et al., 1985; Fernández-Suárez et al., 1998; Leterrier and Noronha, 1998; Valle et al., 1999). The data indicate that by the Early Cambrian, the arc source furnishing the ca. 640-800 Ma zircons had either been eroded/submerged or had drifted away from the platform upon which the Palaeozoic rocks were deposited, or a combination of both.

The ca. 0.9 - 1.2 Ga old zircons from Neoproterozoic and Palaeozoic samples are the first evidence for a Grenvillian age crustal component in NW Iberia, which has important implications for its Neoproterozoic position (see below). The data indicate that Mesoproterozoic zircons found in Neoproterozoic-Lower Palaeozoic sedimentary rocks from NW Iberia have ages that match the span of the Grenville event in Laurentia or the Sunsas/Rondonian event in the Amazon craton (e.g., Nance and Murphy, 1996; Keppie et al., 1998 and references therein). Similar ages have been reported from the Moldanubian zone of the Bohemian Massif and from the French Massif Central (Gebauer et al., 1989). The provenance area of the Grenvillian zircons could be sought either in exposed basement rocks of the Avalonian/Cadomian arc (van Staal et al., 1996; Sheridan et al., 1999) or in a cratonic source located in the margin of Gondwana during the Neoproterozoic/Early Palaeozoic. The proximal character of an igneous Grenvilli an age source is supported by the morphological features of zircons, indicating a short waterborne transport history, and the improbability of their being involved in more than one sedimentary cycle. It is also noteworthy that the Neoproterozoic sedimentary rocks seem to contain rare zircons of pre-Mesoproterozoic age relative to zircons of Grenvillian and Early Cadomian age (Table 1, Fig. 5). This may suggest that the arc contributed more detritus to the basin where Neoproterozoic rocks were deposited than the continental cratonic source. In addition, $\mathrm{Nd}$ isotope signatures of Cadomian granitoids in $\mathrm{NW}$ Iberia intruded into the Neoproterozoic sedimentary rocks at ca. 600 Ma (Fernández-Suárez et al., 1998; Fig. 4) suggest the possible existence of a basement component with a broadly Grenvillian mantle extraction age, a feature frequently found in other Cadomian/Avalonian circum-Atlantic terranes (e.g. Murphy et al., 1996).

Zircons with pre-Mesoproterozoic ages are present in all the sedimentary rocks studied. However, only three concordant analyses were obtained in zircons from samples JG-12, JG-2 and JG-3 (Table 1). These data are consistent with the existence of a Palaeoproterozoic (ca. 1.8-2.0 Ga) crustal component in the basement of NW Iberia (cf. Gebauer, 1993). This age corresponds to the Icartian-Eburnian-Transamazonian events recorded in different terranes of Africa and South America (e.g. Keppie et al., 1998 and references therein). In western Europe, the ca. 1.8-2.1 Ga basement is represented by the Icartian granitic orthogneisses (2061 $\pm 2 \mathrm{Ma}$ ) exposed in the Channel Islands (North Armorican Massif of Cadomia) (Samson and D’Lemos, 1998). Similar ages $(1.8-1.9 \mathrm{Ga})$ were reported from acid granulite boulders collected in the submarine environment of the northern coast of Spain (Guerrot et al., 1989). Gebauer et al. (1989) reported similar ages (ca. $1.85 \mathrm{Ga}$ ) for detrital zircons in high-grade metasedimentary rocks from the Montaigne Noire of the French Massif Central. The presence of ca. 1.8 - 2 Ga zircons in sedimentary rocks from NW Iberia is in agreement with its peri-Gondwanan location during Neoproterozoic-early Palaeozoic times, but can be interpreted in different ways: (i) the zircons are derived from igneous sources like those represented by the Icart gneisses; (ii) they represent inherited xenocrysts in Grenvillian or Early Cadomian igneous rocks; (iii) they are recycled from a post-Icartian sedimentary formation that is no longer exposed. The third possiblity is 
not favoured on the basis of zircon morphology and the features of the host sedimentary rocks (see above). The second possibility also seems improbable given the concordant nature of the analyses. If these zircons were hosted by rocks that suffered high-grade metamorphism and anatexis, they are likely to have suffered $\mathrm{Pb}$ loss. Therefore we suggest that they are likely derived from Icartian-Eburnean-Transmazonian igneous sources. The reported Archaean ${ }^{207} \mathrm{~Pb} /{ }^{206} \mathrm{~Pb}$ ages $(2.5-2.8 \mathrm{Ga})$ match the age span of the Central Amazonian tectonothermal events in the Amazon craton (Teixeira et al., 1989).

\subsection{Insights from comparison of $U-P b$ and $\mathrm{Sm}-\mathrm{Nd}$ data}

Geochemical and Sm- Nd data are available for Neoproterozoic and Palaeozoic metasedimentary rocks from NW Iberia (Beetsma, 1995; Ortega, 1995; Nägler et al., 1995). These data are summarised in Fig. 6 and briefly discussed below in the light of the ages of detrital zircons obtained in this study. The major and trace element signatures of Neoproterozoic rocks in NW Iberia are compatible with an origin in an active margin-continental arc setting (Beetsma, 1995; Nägler et al., 1995). This is consistent with relatively high $\mathrm{S}_{\mathrm{Nd}}$ values $(>-5)$ at the time of deposition and low $T_{\text {DM }}$ values $(1.3-1.6 \mathrm{Ga}$, Fig. 6$)$, that indicate an important contribution of juvenile crust in the Neoproterozoic sedimentary rocks. This is consistent with the ages of detrital zircons reported in this study, which confirm a dominant input of Early Cadomian/Avalonian (ca. 800 - 640 Ma) crust in the Neoproterozoic sedimentary rocks and also a significant input from a likely igneous Grenvillian source. As illustrated in Fig. 6, the $\mathrm{Nd}$ isotopic composition of Neoproterozoic sedimentary rocks is compatible with a dominant input from Cadomian and Grenvillian juvenile crust, plus a minor component of older more enriched sources (Palaeoproterozoic, Archaean).

In contrast, Cambrian sedimentary rocks (even the lowermost Cambrian) have significantly lower $\mathrm{S}_{\mathrm{Nd}}(\mathrm{T})$ values $(<-6)$ and higher $T_{\mathrm{DM}}$ values

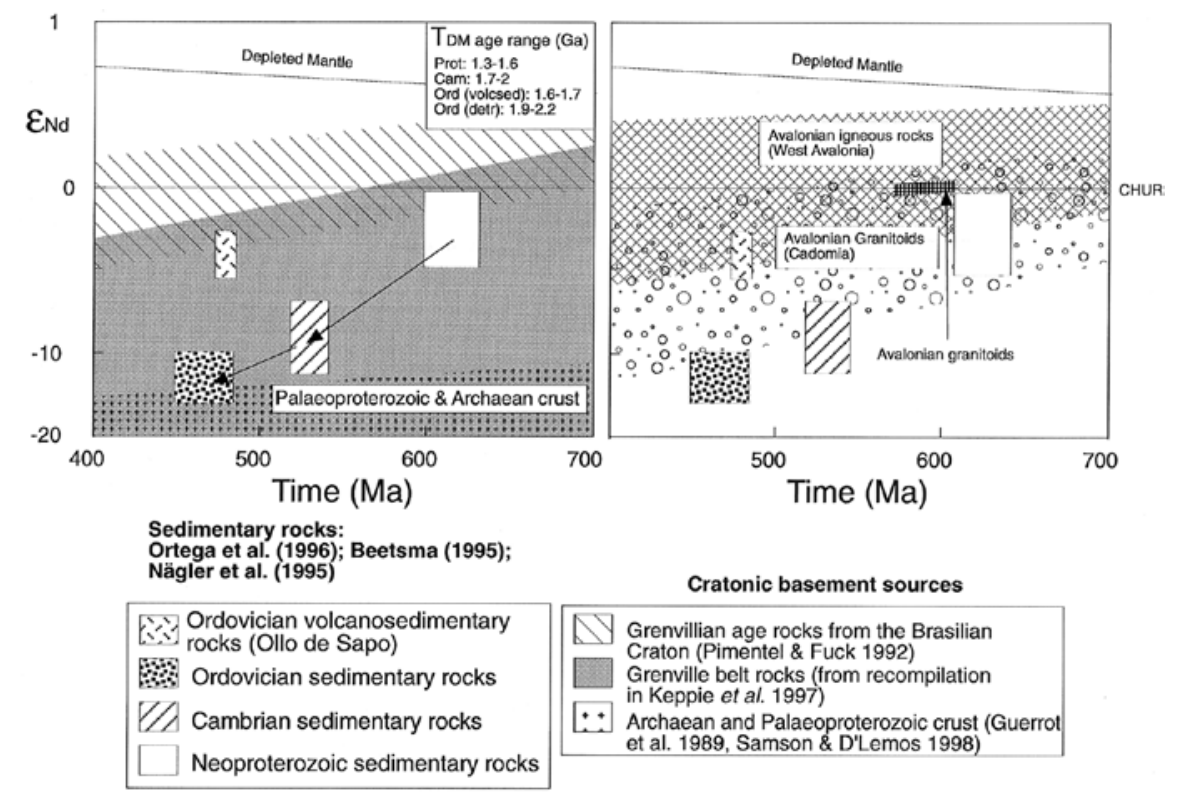

Fig. 6. Plot of $\mathrm{s}_{\mathrm{Nd}}(\mathrm{T})$ versus Age of deposition of Neoproterozoic and early Palaeozoic sedimentary rocks from NW Iberia (sources of analytical data: Beetsma, 1995; Nägler et al., 1995; Ortega, 1995). $T_{\mathrm{DM}}$ values shown as inset have been recalculated using the equation of Liew and Hofmann (1988). For comparison Nd envelopes of Grenvillian, Palaeoproterozoic, and Archaean crust are shown in addition to envelopes for Avalonian granitoids from Iberia (Fernández-Suárez et al., 1998), West Avalonia (Nance and Murphy, 1996; Keppie et al., 1997) and Cadomia (from compilation in Nance and Murphy, 1996). 


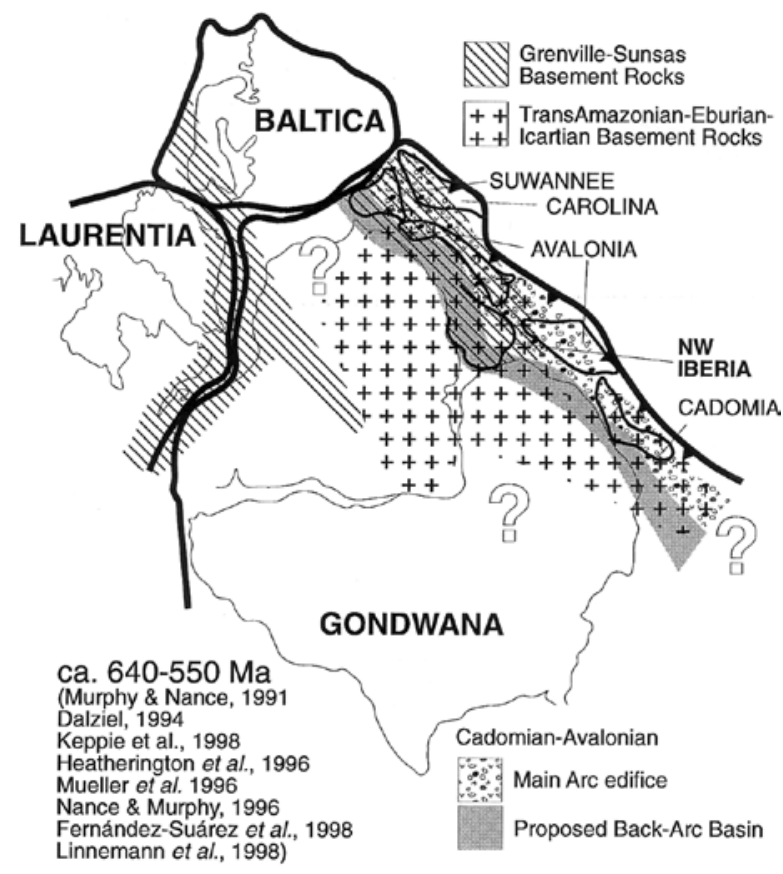

Fig. 7. Proposed paleogeographic reconstruction of Neoproterozoic peri-Gondwanan terranes (based on Nance and Murphy, 1996; Keppie et al., 1998). NW Iberia is considered to have occupied a peri-Amazonian location (see text for discussion) and was possibly situated further towards the continent with respect to West Avalonia.

(1.7-2 Ga, Fig. 6). This change in the Nd isotopic signature is an indication that the contribution of juvenile crust dwindled significantly in a relatively short time span. In the light of zircon ages, this is reflected in the almost complete lack of zircons in the age range $800-640 \mathrm{Ma}$ in the Lower Cambrian sub-arkose (JG-16). Zircons with Cadomian/Avalonian ages in this sample fall in the age range 570-615 Ma, which matches the age range of the scarce Late Cadomian granitoids intruded into the Neoproterozoic sedimentary rocks, and have been interpreted to be generated through lower crustal melting (Fernández-Suárez et al., 1998).

Detrital Ordovician sedimentary rocks (Fig. 6) have even more negative $s_{N d}(T)$ values and higher average $T_{\mathrm{DM}}$ values than the Cambrian sedimentary rocks. This is consistent with a progressively waning contribution of Cadomian/Avalonian newly accreted crust. However, in Ordovician volcano-sedimentary greywackes (JG2, JG3) there is an increase of $\mathrm{S}_{\mathrm{Nd}}(\mathrm{T})$ values and a lowering of $T_{\mathrm{DM}}$ values in relation to an addition of juvenile crust related to the 'Ollo de Sapo' magmatic event (ca. $480-460 \mathrm{Ma}$ ) (see also above). The $\mathrm{U}-\mathrm{Pb}$ zircon ages in samples JG-2 and JG-3 are consistent with $\mathrm{Nd}$ isotopic signatures reported in similar rocks, which could result from a mixture of Palaeoproterozoic (ca. 2 Ga), Mesoproterozoic (ca. 1 Ga) Late-Cadomian (ca. 550 - 620) and Ordovician (Ollo de Sapo) juvenile components.

\subsection{Paleogeographic implications}

A significant implication of the ages obtained in this study concerns the location of NW Iberia in pre-Palaeozoic paleogeographic reconstructions. It is currently accepted that Avalonian/Cadomian circum-Atlantic terranes are dismembered fragments of an accretionary orogenic belt developed on an active margin of Gondwana (e.g. Nance and Murphy, 1996). This peripheral orogen was adjacent to cratonic provinces recording different events of continental assembly, and therefore the Cadomian-Avalonian terranes were most likely developed on different cratonic basements. On the basis of available geological, geochronologic and $\mathrm{Nd}$ isotopic signatures of these terranes it has been considered so far that West Avalonia, Carolina and Florida were situated in the periphery of the Amazonian craton during the Neoproterozoic, whereas Cadomia, Iberia and portions of East Avalonia are thought to have laid adjacent to (and evolved upon) the West African cratonic crust at that time (e.g. Krö ner et al., 1988; Guerrot et al., 1989; Dalziel, 1992; Nance and Murphy, 1996; Keppie et al., 1998).

The presence of seemingly proximal GrenvilleSunsas/Rondonian age zircons in Neoproterozoic sedimentary rocks from NW Iberia argues against a peri-West African location during the Neoproterozoic, unless a yet unidentified Grenvillian event is also present within the West African craton. With the evidence available to date, the data reported in this study suggest a peri-Amazonian location of Iberia closer to West Avalonia, Florida and Carolina during the Neoproterozoic, as tentatively depicted in Fig. 7. In addition, the few discordant zircons with ${ }^{207} \mathrm{~Pb} /{ }^{206} \mathrm{~Pb}$ ages 
around 1.4-1.6 Ga (Table 1, Fig. 5) could have their provenance in the Rio Negro orogen of the Amazon craton (cf. Keppie et al., 1998), an orogenic event that is not recorded in the West African craton. Furthermore, the fact that Neoproterozoic NW Iberia mainly records back-arc basin sedimentation and relatively scarce magmatic activity (cf. Fernández-Suárez et al., 1998) suggests a continentwards location such as that depicted in Fig. 7. The hypothesis of a peri-Amazonian location of Iberia in Neoproterozoic times is strengthened by its position after undoing the Alpine and late/post Variscan rotations, which suggests a more north westerly paleo-location of this area with respect to Cadomia and East Avalonia.

Available Sm- Nd data on ca. 600 My old granitoids from the Narcea Antiform (Fig. 6) $\left(\mathrm{S}_{\mathrm{Nd}} \geqslant 0, T_{\mathrm{DM}} \geqslant 1.2 \mathrm{Ga}\right.$, Fernández-Suárez et al., 1998) are compatible with Sm- Nd signatures of Avalonian granitoids from terranes considered to have evolved upon Amazonian cratonic basement (cf. Nance and Murphy, 1996). The Nd isotopic data from those Avalonian/Cadomian granitoids of NW Iberia, taken together with detrital zircon ages (this study) suggest that NW Iberia might have evolved upon a basement containing a significant component of Grenvillian age crust and occupied a location close to South America during the Neoproterozoic where it would have had access to detrital zircons from the Amazon craton.

The Palaeoproterozoic (Eburnian-IcartianTransamazonian) ages obtained (ca. 1.8-2 Ga) are consistent with the peri-Gondwanic nature of Iberia and the other terranes mentioned above since ca. 1.9-2.1 Ga events are recorded in both the West African craton (Eburnian event) and the Amazon craton (Trans-Amazonian event). The data also confirm the involvement of Archaean crustal components in the Neoproterozoic and subsequent events in NW Iberia (cf. Guerrot et al., 1989; Gebauer, 1993). The apparent absence of zircons with ${ }^{207} \mathrm{~Pb} /{ }^{206} \mathrm{~Pb}$ ages older than ca. $2.8 \mathrm{Ga}$ could also be an indication that NW Iberia did not occupy a peri-west African location during the Neoproterozoic, as the Archaean crust in the West African craton records Archaean tectonothermal events older than ca. 2.7 Ga (Rocci et al., 1991).

\subsection{Tentative scenario of Proterozoic-Early Palaeozoic in NW Iberia}

Fig. 8 depicts the tentative scenario proposed for the Neoproterozoic-early Palaeozoic evolution of NW Iberia. The data reported here and available $\mathrm{U}-\mathrm{Pb}$ and $\mathrm{Nd}$ isotope data from other areas point to the existence of a complex cratonic basement in the European Variscides including Archaean, Palaeoproterozoic and Mesoproterozoic components (Liew and Hofmann, 1988; Guerrot et al., 1989; Pin and Duthou, 1990; Turpin et al., 1990; Downes et al., 1990; Gebauer, 1993; Beetsma, 1995; Nägler et al., 1995; Samson and D’Lemos, 1998; Zeck and Whitehouse, 1999). However, actual contributions of the proposed crustal components to the basement of pre-Neoproterozoic Iberia and other areas of the European Variscides are poorly constrained. Our contention, based on the abundance of Grenvillian age zircons and model ages of Cadomian granitoids in NW Iberia is that the pre-Neoproterozoic basement might have had a significant Grenvillian crustal component.

The pre-Neoproterozoic basement (Fig. 8a) is taken as a starting point for the proposed scenario of Neoproterozoic-early Palaeozoic evolution of NW Iberia. Reconstructions of Gondwana during Late Proterozoic times show a peripheral arc edifice related to an oblique subduction event on its margin (Fig. 8b). The magmatic activity along this arc (Fig. 8b, c) is thought to have reached its climax between ca. 640 and 580 Ma (Murphy and Nance, 1991; Nance et al., 1991; Nance and Murphy, 1996; Keppie et al., 1998) although magmatic activity related to arc construction is considered to have started some $100-$ 150 Ma before (Nance, 1990; Murphy and Nance, 1991; Egal et al., 1996; O’Brien et al., 1996). Back arc basins were developed during this subduction event (Nance et al., 1991; Murphy and Nance, 1991; Nance and Murphy, 1996; FernándezSuárez et al., 1998) and the Neoproterozoic sediments are thought to have been deposited in these basins and to derive mainly from the arc (Murphy and Nance, 1989, 1991).

Fig. 8c depicts the continuation of subduction and arc-related magmatism down to ca. $560 \mathrm{Ma}$. 
The back arc basin shown in this figure is considered to represent the locus of deposition for the Neoproterozoic sedimentary rocks of NW Iberia (Fernández-Suárez et al., 1998). It is proposed that these rocks contain detritus from both the main arc edifice and the margin of Gondwana. The presence of detrital components from the main arc edifice is represented by the ca. $640-800$ Ma zircon population found in the Neoproterozoic rocks. This is in agreement with $\mathrm{Nd}$ isotopic signatures that indicate a significant component of newly accreted crust in these sedimentary rocks (see Section 6.2). The Grenvillian age zircons $(0.9-1.27 \mathrm{Ga})$ may have reached this basin(s)
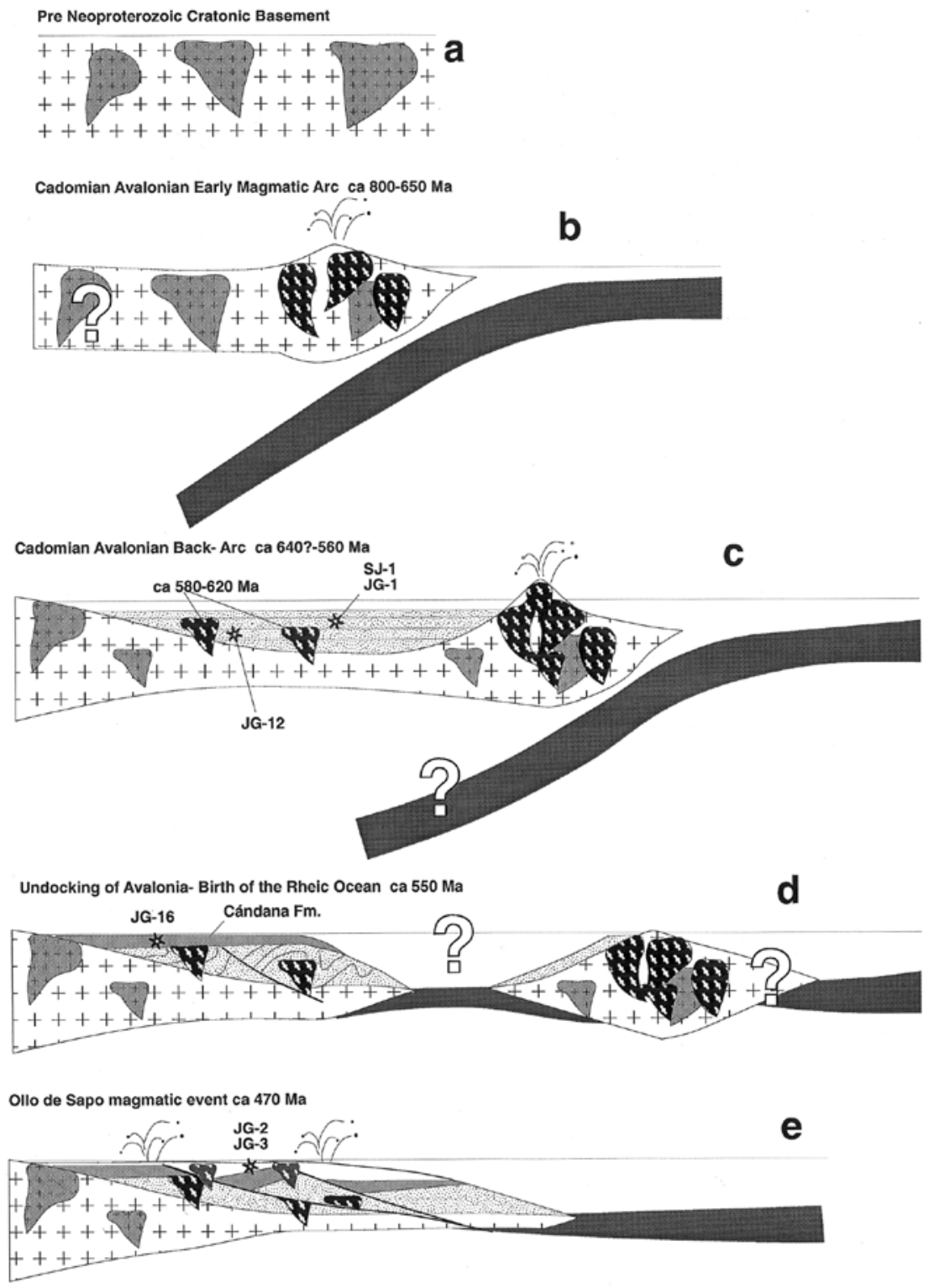

Fig. 8. Proposed schematic scenario for the Proterozoic-early Palaeozoic geological evolution of NW Iberia. See full discussion in text. 
either from exposed fragments of the arc basement or from the continentwards cratonic source (see Section 6.3 above). The data suggest that the most likely cratonic source is the Sunsas/Rondonian crust found in the Amazonian craton of Gondwana. Older zircons (Lower Proterozoic and Archaean) would also have their source in their counterpart cratonic portions of Amazonia. The latter is in agreement with geochemical data suggesting the involvement of a stable cratonic source in the genesis of the Neoproterozoic sedimentary rocks from the Spanish Central Iberian Zone (Ugidos et al., 1997).

In NW Iberia, as in other areas of the Avalonian/Cadomian belt (cf. Murphy and Nance, 1991) geological evidence (e.g. lack of widespread deformation or significant crustal thickening) suggests that the termination of the subduction event was not due to final continent- continent collision. We concur with the views of Murphy and Nance (1989) who proposed that the subduction was replaced by transform/extensional activity related to the breakup of the late Precambrian supercontinent. Based upon the data from NW Iberia, our hypothesis is that following the arrest of subduction or a change in subduction regime (from ridge push to slab pull?), further extension of the basin resulted in the undocking of Avalonia (main arc edifice) from Gondwana, the opening of the Rheic ocean and eventually, the genesis of the Iberian-Armorican and Meguma platforms on both sides of the ocean (Fig. 8d) (see also Fortey et al., 1988; Soper, 1988). This event is reflected in the scarcity of ca. 640-800 Ma detrital zircons in the Lower Cambrian Cándana Formation (JG16), that lies unconformably over the Neoproterozoic sequence. The Cándana Formation does contain abundant late Cadomian zircons (550 $620 \mathrm{Ma}$ ) likely recycled through exhumation and erosion of the granitoids intruded into the Neoproterozoic sediments of NW Iberia (Fig. 8c, d). The Grenvillian age zircons and those with pre-Mesoproterozoic ages could have their source either in the cratonic areas of Gondwana or in the Neoproterozoic sedimentary rocks, which already contain these age populations. However, the morphological features of zircons suggest that the latter case is unlikely. Available $\mathrm{Nd}$ isotopic data
(Fig. 6) suggest that the actual contribution of juvenile crust is not very significant in Cambrian rocks, in spite of the seeming abundance of ca. 550-620 Ma zircons (Fig. 4d).

In the Ordovician, it is proposed that further extension of an already thinned crust (Fig. 8e) gave rise to the magmatic Ollo de Sapo event (ca. $460-490 \mathrm{Ma}$ ) in Iberia, likely to have been triggered by mantle upwelling and upper mantlelower crustal melting in a progressively thinning lithosphere (Fernández-Suárez et al., 1998). The genesis of these rocks in an extensional intracontinental setting is consistent with the within-plate trace element signatures of coeval alkalic basaltic rocks (Loeschke and Zeidler, 1982; Valverde-Vaquero, 1992) and the presence of Early Ordovician peralkaline granitoids in the margin of Gondwana (Van Calsteren et al., 1979). In the Ollo de Sapo greywackes, the juvenile volcanic component is reflected both directly by the $440-$ 460 Ma zircon population and higher $\mathrm{s}_{\mathrm{Nd}}$ values and younger $T_{\mathrm{DM}}$ ages with respect to Ordovician detrital (no volcanic components) sediments. As stated earlier, these samples contain Cadomian, Grenvillian and Palaeoproterozoic zircons from a proximal Gondwanan source. In NW Iberia, the oldest $T_{\mathrm{DM}}$ ages and maximum spread of $T_{\mathrm{DM}}$ values in sedimentary rocks correspond to an age of ca. 440-470 Ma (i.e. overlapping and following the Ollo de Sapo event). The combined $T_{\mathrm{DM}}$ and $\mathrm{S}_{\mathrm{Nd}}$ spread (Beetsma, 1995; Nägler et al., 1995) reflect both an increase in the contribution from older crustal components and the addition of newly accreted crust. We suggest that this increase in addition of older material could be related to the genesis of the OS rocks in a crust undergoing progressive extension (see above). A progressively thinning crust is a likely scenario that would explain the simultaneous exhumation of lower crustal material and the generation of coeval magmatism. This latter scenario is consistent with models proposed for other circum-North Atlantic Avalonian/Cadomian terranes where repeated episodes of melting occurred in response to subduction and subsequent rifting events (Murphy et al., 1996; Mancuso et al., 1996). This scenario is also in agreement with current models for the subsequent evolution of Iberia from Silurian to 
Late Carboniferous times (e.g. Martínez Catalán et al., 1997).

\section{Acknowledgements}

J. F-S wishes to acknowledge a post-doctoral grant from the Spanish Ministry of Education. Financial support for this research was provided by an NSERC operating grant to GAJ, an NSERC Major Facilities Access Grant for the ICP-MS facility (Memorial University) and a NATO travel grant to G.A. Jenner, F. Bea and J. Fernández-Suárez. Financial support to G. Gutiérrez-Alonso was provided by DGIYCT project PB96-1452-C03-02. Discussions with P. Sylvester helped to improve and clarify parts of this paper. Thorough and stimulating reviews by J.B. Murphy, J.D. Keppie, S. Samson and A. Kröner are kindly acknowledged.

\section{References}

Bastida, F., Marcos, A., Marquínez, J., Martínez Catalán, J.R., Pérez Estaún, A., Pulgar, J.A., 1984. Mapa Geológico de España E 1:200.000 1(2-1) La Coruña. Instituto Geológico y Minero de España, 155 pp.

Beetsma, J.J., 1995. The late Proterozoic/Paleozoic and Hercynian crustal evolution of the Iberian Massif, N Portugal, as traced by geochemistry and $\mathrm{Sr}-\mathrm{Nd}-\mathrm{Pb}$ isotope systematics of pre-Hercynian terrigenous sediments and Hercynian Granitoids. PhD Thesis, Vrije Universiteit, Amsterdam, The Nederlands, 223 pp.

van den Bosch, W.J., 1969. Geology of the Luna-Sil region, Cantabrian Mountains (NW Spain). Leidse Geologische Mededlingen 44, 137-225.

Calvez, J.Y., Vidal, Ph., 1978. Two billion years old relicts in the Hercynian Belt of Western Europe. Contrib. Mineralol. Petrol. 65, 395-399.

Capdevila, R., 1969. Le metamorphisme regional progressif et les granites dans le segment hercynien du NW de l'Espagne. PhD Thesis, University Montpellier, France, 430 pp.

Crimes, T.P., Legg, I., Marcos, A., et al., 1977. Late precambrian-low Lower Cambrian trace fossils from Spain. In: Crimes, T.P., Harper, J.C. (Eds.), Trace fossils. Geol. J. Spec. Issue 9, 91-138.

Dallmeyer, R.D., Martínez Catalán, J.R., Arenas, R., et al., 1997. Diachronous Variscan tectonothermal activity in the NW Iberian Massif: evidence from 40Ar/39Ar dating of regional fabrics. Tectonophysics 277, 307-337.
Dalziel, I., 1992. On the organization of American plates in the Neoproterozoic and the breakout of Laurentia. GSA Today 2, 238-241.

Downes, H., Dupuy, C., Leyreloup, A.F., 1990. Crustal evolution of the Hercynian belt of western Europe: evidence from lower-crustal granulitic xenoliths (French Massif Central). Chem. Geol. 83, 209-231.

Egal, E., Guerrot, C., Le Goff, E., et al., 1996. The Cadomian orogeny revisited in northern Brittany (France). In: Nance, R.D., Thompson, M.D. (Eds.), Avalonian and Related Peri-Gondwanan Terranes of the Circum-North Atlantic. Boulder, CO. Geol. Soc. Am. Spec. Paper 304, 281-318.

Fernández-Suárez, J., Gutiérrez-Alonso, G., Jenner, J.A., et al., 1998. Geochronology and geochemistry of the Pola de Allande granitoids (northern Spain). Their bearing on the Cadomian/Avalonian evolution of NW Iberia. Can. J. Earth Sci. 35, 1439-1453.

Fernández-Suárez, J., Gutiérrez-Alonso, G., Jenner, J.A., et al., 1999. Crustal sources in Lower Palaeozoic rocks from NW Iberia: insights from laser ablation $\mathrm{U}-\mathrm{Pb}$ ages of detrital zircons. J. Geol. Soc. London 156, 1065-1068.

Gebauer, D., 1993. Intra-grain zircon dating within the Iberian Massif: Ollo de Sapo augengneisses, bimodal gneisses from the Massif de Guilleries (Girona), Graywacke of the Tentudía Group (serie Negra, SW Spain) and the HP/HT-rock association at Cabo Ortegal (Galicia). XII Reunião de Geologia do Oeste Peninsular. Comunicaçõ es, Evora, Portugal, 41-46.

Gebauer, D., Williams, I.S., Compston, W., et al., 1989. The development of the Central European continental crust since the Early archean based on conventional and ion-microprobe dating of up to 4.84 b.y. old detrital zircons. Tectonophysics 157, 81-96.

Guerrot, C., Peucat, J.J., Capdevila, R., 1989. Archean protoliths within Early Proterozoic granulitic crus of the west European Hercynian belt: possible relics of the west African craton. Geology 17, 241-244.

Gutiérrez-Alonso, G., Fernández-Suárez, J., 1996. Geología y geoquímica del granitoide pre-Varisco de Puente de Selce (Antiforme del Narcea, Asturias). Revista de la Sociedad Geológica de España 9, 227-239.

Hirata, T., Nesbitt, R.W., 1995. U- Pb isotope geochronology of zircon: evaluation of the laser probe-inductively coupled plasma mass spectrometry technique. Geochim. Cosmochim. Acta 59, 2491-2500.

Ireland, T.R., Flottmann, T., Fanning, C.M., et al., 1998. Development of the early Paleozoic Pacific margin of Gondwana from detrital-zircon ages across the Delamerian orogen. Geology 26, 243-246.

Jackson S.E., Longerich, H.P., Horn, I., Dunning, G., 1997. The application of laser ablation microprobe (LAM)-ICPMS to in situ $\mathrm{U}-\mathrm{Pb}$ zircon geochronology, Proceedings of the Goldschmidt Conference, 283-284.

Keppie, J.D., Dostal, J., Murphy, J.B., Cousens, B.L., 1997. Palaeozoic within-plate volcanic rocks in Nova Scotia (Canada) reinterpreted: isotopic constraints on magmatic source and paleocontinental reconstructions. 
Keppie, J.D., Davis, D.W., Krogh, T.E., 1998. U- Pb geochronological constraints on Precambrian stratified units in the Avalon Composite Terrane of Nova Scotia, Canada: tectonic implications. Can. J. Earth Sci. 35, 222 236.

Krö ner, A., Wendt, I., Compston, W., et al., 1988. U- Pb zircon and Sm- Nd model ages of high grade Moldanubian metasediments, Bohemian Massif, Czechoslovakia. Contrib. Mineral. Petrol. 99, 257-266.

Lancelot, J.R., Allegret, A., Iglesias, M., 1985. Outline of upper Precambrian and Lower Paleozoic evolution of the Iberian Peninsula according to $\mathrm{U}-\mathrm{Pb}$ dating of zircons. Earth Planetary Sci. Lett. 74, 325-337.

Leterrier, J., Noronha, F., 1998. Os gnaisses pre-Hercínicos a Hercínicos precoces da Serra de Vila Mendo (Sul de Penalba do Castelo) - ZCI: Caracterizaçao estrutural e geoquímica.

Liew, T.C., Hofmann, A.W., 1988. Precambrian crustal components, plutonic associations, plate environment of the Hercynian foldbelt of central Europe: implications from a Nd and Sr isotopic study. Contrib. Mineral. Petrol. 98, 129-138.

Loeschke, W.H.J., Zeidler, N., 1982. Early Paleozoic sills in the Cantabrian Mountains (Spain) and their geotectonic environment. Neues Jahrb. Geol. Palaontol. Monatash 7, 419-439.

Martínez Catalán, J.R., Arenas, R., Díaz García, F., et al., 1996. Variscan exhumation of a subducted Paleozoic continental margin: the basal units of the Ordenes complex, Galicia, NW Spain. Tectonics 15, 106-121.

Martínez Catalán, J.R., Arenas, R., Díaz García, F., et al., 1997. Variscan accretonary complex of northwest Iberia: Terrane correlation and succession of tectonothermal events. Geology 25, 1103-1106.

Moreno-Ventas, I., Rogers, G., Castro, A., 1995. The role of hybridization in the genesis of hercynian granitoids in the Gredos Massif, Spain: inferences from $\mathrm{Sr}-\mathrm{Nd}$ isotopes. Contrib. Mineral. Petrol. 120, 137-149.

Murphy, J.B., Nance, R.D., 1989. Model for the evolution of the Avalonian-Cadomian belt. Geology 17, 735-738.

Murphy, J.B., Nance, R.D., 1991. Supercontinent model for the contrasting character of Late Proterozoic orogenic belts. Geology 19, 469-472.

Murphy, J.B., Keppie, J.D., Dostal, J., et al., 1996. Repeated late Neoproterozoic-Silurian lower crustal melting beneath the Antigonish Highlands, Nova Scotia: Nd isotopic evidence and tectonic interpretations. In: Nance, R.D., Thompson, R.D. (Eds.), Avalonian and Related PeriGondwanan Terranes of the Circum-North Atlantic. Boulder, CO. Geol. Soc. Am. Spec. Paper 304, 109-120.

Nägler, T.F., Schäfer, H.J., Gebauer, D., 1995. Evolution of the Western Europesn continental crust: implications from $\mathrm{Nd}$ and $\mathrm{Pb}$ isotopes in Iberian sediments. Chem. Geol. 121, 345-347.

Nance, R.D., 1990. Late Precambrian-Early Palaeozoic evolution of part of the Avalon terrane in southern New Brunswick. In: D'Lemos, R.S., Strachan, R.S., Topley,
C.G. (Eds.), The Cadomian orogeny. Geol. Soc. Spec. Publ. 51, 363-382.

Nance, R.D., Murphy, J.B., 1996. Basement isotopic signatures and Neoproterozoic paleogeography of AvalonianCadomian and related terranes in the Circum-North Atlantic. In: Nance, R.D., Thompson, M.D. (Eds.), Avalonian and Related Peri-Gondwanan Terranes of the Circum-North Atlantic. Boulder, CO. Geol. Soc. Am. Spec. Paper 304, 109-120.

Nance, R.D., Murphy, J.B., Strachan, R.A., et al., 1991. Late Proterozoic tectonostratigraphic evolution of the Avalonian and Cadomian terranes. Precambrian Geol. 53, 41 78.

O'Brien, S.J., O'Brien, B.H., Dunning, G.R., et al., 1996. Late Neoproterozoic Avalonian and related peri-Gondwanan rocks of the Newfoundland Appalachians. In: Nance, R.D., Thompson, M.D. (Eds.), Avalonian and Related Peri-Gondwanan Terranes of the Circum-North Atlantic. Boulder, CO. Geol. Soc. Am. Spec. Paper 304, 9-28.

Ortega, L.A., 1995. Estudio petrogenético del granito sincinemático de dos micas de La Espenuca (La Coruña). Ph D Theis. Univerdidad del Pais Vasco, 367 pp.

Ortega, L.A., Carracedo, M., Larrea, F.J., Gil Ibarguchi, J.I., 1996. Geochemistry and tectonic enviroment of Volcanosedimentary rocks from the Ollo de Sapo Formation (Iberian Massif, Spain). In: Demaiffe, D. (Ed.), Petrology and Geochemistry of magmatic suites of rocks in the continental and oceanic crusts. A volume dedicated to Professor Jean Michot. Université Libre de Bruxelles. Royal Museum for Central Africa (Tervuren). 277-290.

Pérez-Estaú n, A., Martínez-Catalán, J.R., Bastida, F., 1991. Crustal thickening and deformation sequence in the footwall to the suture of the Variscan Belt of NW Spain. Tectonophysics 191, 243-253.

Pin, C., Duthou, J.L., 1990. Sources of Hercynian granitoids from the Grench Masif Central: inferences from Nd isotopes and consequences for crustal evolution. Chem. Geol. 83, 281-296.

Rainbird, R.H., McNicoll, V.J., Thériault, R.J., et al., 1997. Pan-continental river system draining Grenville orogen recorded by $\mathrm{U}-\mathrm{Pb}$ and and $\mathrm{Sm}-\mathrm{Nd}$ geochronology of Neoproterozoic quartzarenites and mudrocks, Northwestern Canada. J. Geol. 105, 1-17.

Samson, S.D., D'Lemos, R.S., 1998. U-Pb geochronology and $\mathrm{Sm}-\mathrm{Nd}$ isotopic composition of Proterozoic gneises, Channel Islands, UK. J. Geol. Soc. London 155, 609-618.

Sheridan, R.E., Maguire, T.J., Feigenson, M.D., et al., 1999. Grenville age basement rocks in cape may nj well: new evidence for laurentian crust in US atlantic coastal plain basement chesapeake terrane. J. Geodynam. 27, 623-633.

Soper, N.J., 1988. Timing and geometry of collision, terrane accretion and sinistral strike-slip events in the British Caledonides. In: Harris, A.L., Fettes, D.J. (Eds.). The Caledonian-Appalachian Orogen. Geological Society of London Special Publication 38, 481-492.

Teixeira, W., Tassinari, C.C.G., Cordani, U.G., et al., 1989. A review of the geochronology of the Amazon craton: tectonic implications. Precambr. Res. 42, 213-227. 
Turpin, L., Cuney, M., Friedrich, M., et al., 1990. Meta-igneous origin of Hercynian peraluminous granites in N.W. French Massif Central: implications for crustal history reconstructions. Contrib. Mineral. Petrol. 104, 163-172.

Ugidos, J.M., Armenteros, I., Barba, P., et al., 1997. Geochemistry of recycled orogen-derived sediments: a case study from Upper Precambrian siliciclastic rocks of the Central Iberian Zone, Iberian Massif, Spain. Precambr. Res. 84, 163-180.

Valle, B., Cordani, U.G., Nolan, J., et al., 1999. U- Pb (SHRIMP) geochronology of pre-Hercynian granitoids of the Iberian Variscan foldbelt (Northern Central Portugal): evidence for a Cadomian magmatic event. Terra Abstr. 11, 110.

Valverde-Vaquero, P., 1992. Permo-Carboniferous magmatic activity in the Cantabrian Zone (NE Iberian Massif, Asturias, NW Spain). MSc Thesis, Boston College.

Valverde-Vaquero, P., Dunning, G.R., 1997. Magmatismo 'Sárdico' Arenig en el Dominio del Ollo de Sapo de la Zona Centro Ibérica: Nuevas evidencias U-Pb en la Sierra de Guadarrama. In XIV Reunião de Geologia do Oeste
Peninsular. Evolução Geoló gica do Maciço Ibérico e seu enquadramento continental. Comunicaçõ es, Vila Real, Portugal. 265-270.

Valverde-Vaquero, P., Dunning, G.R., 1999. New U- Pb ages for Early Ordovician Magmatism in Central Spain. J. Geol. Soc. London (in the press).

van Staal, C.R., Sullivan, R.W., Whalen, J.B., 1996. Provenance and tectonic history of the Gander Zone in the Caledonian/Appalachian orogen: implications for the origin and assembly of Avalon. In: Nance, R.D., Thompson, M.D. (Eds.), Avalonian and Related Peri-Gondwanan Terranes of the Circum-North Atlantic. Boulder, CO. Geol. Soc. Am. Spec. Paper 304, 347-367.

Vidal, G., Palacios, T., Gámez-Vintaned, J.A., et al., 1994. Neoproterozoic-early Cambrian geology and palaeontology of Iberia. Geol. Magazine 131, 729-765.

Zeck, H.P., Whitehouse, M.J., 1999. Hercynian, Pan-African, Proterozoic and Archean ion-microprobe zircon ages for a Betic-Rif core complex, Alpine belt, W Mediterranean consequences for its P-T-t path. Contrib. Mineral. Petrol. 134, 134-149. 